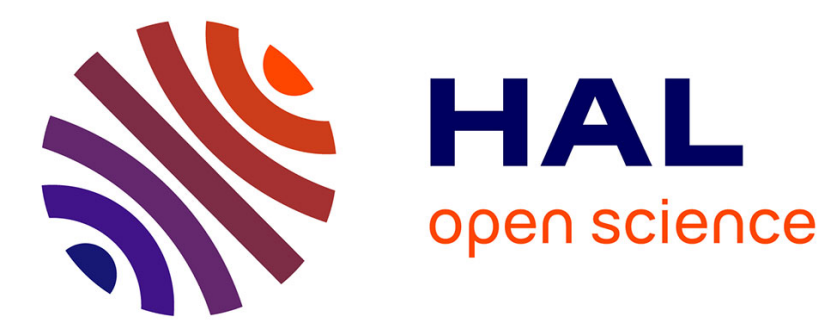

\title{
Seismic surveying and imaging at the laboratory scale: A framework to cross-validate experiments and simulations for a salt-body environment
}

Bence Solymosi, Nathalie Favretto-Cristini, Vadim Monteiller, Paul Cristini, Bjørn Ursin, Dimitri Komatitsch

\section{To cite this version:}

Bence Solymosi, Nathalie Favretto-Cristini, Vadim Monteiller, Paul Cristini, Bjørn Ursin, et al.. Seismic surveying and imaging at the laboratory scale: A framework to cross-validate experiments and simulations for a salt-body environment. Geophysics, 2020, 85 (3), pp.T123-T139. 10.1190/geo20190313.1. hal-03023394

\section{HAL Id: hal-03023394 https://hal.science/hal-03023394}

Submitted on 25 Nov 2020

HAL is a multi-disciplinary open access archive for the deposit and dissemination of scientific research documents, whether they are published or not. The documents may come from teaching and research institutions in France or abroad, or from public or private research centers.
L'archive ouverte pluridisciplinaire HAL, est destinée au dépôt et à la diffusion de documents scientifiques de niveau recherche, publiés ou non, émanant des établissements d'enseignement et de recherche français ou étrangers, des laboratoires publics ou privés. 


\title{
Seismic surveying and imaging at the laboratory scale: A framework to cross-validate experiments and simulations for a salt-body environment
}

\author{
Bence Solymosi ${ }^{1}$, Nathalie Favretto-Cristini ${ }^{1}$, Vadim Monteiller ${ }^{1}$, Paul Cristini ${ }^{1}$, Bjørn \\ Ursin $^{2}$, and Dimitri Komatitsch ${ }^{1}$
}

\begin{abstract}
${ }^{1}$ Aix-Marseille Univ, CNRS, Centrale Marseille, LMA, Marseille, France. E-mail: bence.solymosi@gmail.com (corresponding author); favretto@Ima.cnrs-mrs.fr; monteiller@Ima.cnrs-mrs.fr; cristini@Ima.cnrs-mrs.fr ${ }^{2}$ NTNU, Department of Electronic Systems, Trondheim, Norway. E-mail: bjorn.ursin@ntnu.no
\end{abstract}

\section{ABSTRACT}

Laboratory experiments have been recently reintroduced into the ideas-to-applications pipeline for geophysical applications. Benefiting from recent technological advances, we believe that in the coming years, laboratory experiments can play a major role in supporting field experiments and numerical modeling, to explore some of the current challenges of seismic imaging in terms of, for instance, acquisition design or benchmarking of new imaging techniques at a low cost and in an agile way. But having confidence in the quality and accuracy of the experimental data obtained in a complex configuration which mimics at a reduced scale a real geologic environment, is an essential prerequisite. This requires a robust framework regardless of the configuration studied. Our goal is to provide a global overview of this framework in the context of offshore seismics. To illustrate it, a reduced-scale model is used to represent a 3D complex-shaped salt body buried in sedimentary layers with curved surfaces. Zero-offset and offset reflection data are collected in a water tank, using a conventional pulse-echo technique. Then a cross-validation approach is applied, which allows through the comparison between the experimental data and the numerical simulation, to point out both some necessary future improvements of the laboratory setup to increase the accuracy of the experimental data, and the limitations of the numerical implementation which must also be tackled. Due to this approach, a hierarchical list of points can be collected, to which particular attention should be paid in order to make laboratory experiments an efficient tool in seismic exploration. Finally, the quality of both the complex reduced-scale model and the global framework is successfully validated by applying reverse-time migration to the laboratory data.

\section{INTRODUCTION}

Numerical simulations of wave propagation are key tools, for example, to help the understanding of field data, in seismic acquisition design, imaging, and inversion during subsurface exploration (Robertsson et al., 2007, Virieux et al., 2011). However, in highly challenging geological environments, for which reliable real seismic datasets are often expensive, incomplete, or simply difficult to obtain due to practical reasons, relying only on a fully numerical approach to study and improve surveying and imaging may be inappropriate, as the numerical datasets usually suffer from simplified physics. Indeed, for instance, they do not contain source-related noise, or often rely on "unphysical" attenuation parameters, such as the quality factor. In this context, after having experienced a drop in laboratory experimentation related papers for two decades, laboratory experiments are now re-considered as a tool to understand real field data and purely numerical datasets as well, to facilitate the testing of new ideas (Becker et al., 2018), to investigate the physics 
underlying wave propagation that is not sufficiently understood (Cooper et al., 2010, Stewart et al., 2012, Ekanem et al., 2013, Xu et al., 2016, Chang et al., 2017), as well as to test numerical algorithms used for data processing and imaging (Campman et al., 2005, Chai et al., 2015). Recently, small-scale modeling approaches have been developed as tools to test numerical modeling and seismic-imaging methods in the context of onshore and offshore seismics (Bretaudeau et al., 2011, 2013, FavrettoCristini et al., 2014, Tantsereva et al., 2014a,b, Solymosi et al., 2018). In particular, Tantsereva et al. (2014a) have evaluated the ability of a 3D discretized Kirchhoff integral method (DKIM) to accurately simulate complex diffractions using a zero-offset laboratory data set, measured for a reduced-scale model with strong topography and immersed in a water tank. Comparisons of numerical and laboratory data sets have shown that the DKIM could correctly reproduce the wavefield, except in the vicinity of shadow zones created by the interaction on the edges of the topographic structures. More importantly, a quantitative analysis of the effect of multiple scattering and the surface curvature on the wavefield has been then performed to define the cases where these effects may be neglected in the numerical modeling, in order to decrease the computational cost while maintaining a high accuracy (Favretto-Cristini et al., 2017). These works clearly show the importance of laboratory experiments as part of the benchmarking options for numerical algorithms. Indeed, reduced-scale experiments are highly valuable because they are repeatable, more controllable than real seismic surveys, and versatile in terms of the acquisition setup. Laboratory experiments also provide data of higher fidelity than real seismic surveys, because the sources and the level of noise, as well as the uncertainties in the transducer positions and in the material properties, can be better assessed than in usual seismic exploration setups.

However, using laboratory experiments to investigate seismic issues raises legitimate concerns. For instance, the issue of similarity is at the heart of the debate between those who support the all numerical approach and those who push for a fine balance between numerical simulations and laboratory experiments. Using laboratory experiments implicitly assumes that the scaled physical mechanisms are identical to those at the seismic scale (Ebrom and McDonald 1994). In other words, waves have to propagate identically in both settings. This implicitly assumes that the sources are able to generate the appropriate wave phenomena, that the geometry of the reduced-scale model is similar (at a smaller scale) to that of the real field, and that the materials used for manufacturing this model behave identically to the real geological media. It is obvious that the size of the sources and receivers used in the laboratory experiments cannot be compared to the sources/receivers used on the field. Nevertheless, laboratory sensors with a large area of illumination to emphasize the different wave phenomena observed in field experiments (e.g., multiple diffractions, wave focusing/defocusing) are available nowadays (Tantsereva et al., 2014a). Moreover, thanks to a broad range of metals, resins, and composite materials, one can often find materials with somewhat identical density and P- and Swave velocities. By applying pre-stressed conditions or by combining various materials, one can even create models with specific anisotropic properties. Nonetheless, scaling experiments in certain geological environments are still challenging. Indeed, as the real-life dimensions are often scaled down to the laboratory scale by a factor of 10000 - 20000 (Bretaudeau et al., 2011, Solymosi et al., 2018), it may be technically difficult to reproduce at a reduced scale some of the heterogeneities found in real geological environments. As a consequence, it is not straightforward to study, for instance, the impact of the non-linear behavior of materials due to micro-damage or cracks on wave propagation. A laboratory for immersive wave experimentations that enables experiments with broadband signals at much lower frequencies than commonly employed in typical wave propagation laboratories is therefore of great interest (Vasmel et al., 2013). However, additive and subtractive manufacturing 
techniques, as well as new composite materials, make it possible to manufacture reduced-scale models that are increasingly realistic from a geological point of view, as illustrated thereafter in this paper. We are convinced that these complex models, associated with a very high quality and accurate laboratory experimental framework, could play an increasingly important role in the exploration of the current challenges of seismic imaging in terms of acquisition design, or in benchmarking new imaging techniques at a low cost and in an agile way. But having confidence in the quality and accuracy of the experimental data obtained in a very complex environment (i.e., an environment that is far beyond the configuration of a flat stratified medium) is an essential prerequisite. As a consequence, this requires a robust framework regardless of the configuration studied.

The goal of this paper is to provide a global overview of the framework to be implemented to obtain high-quality laboratory data in the context of offshore seismics. The framework is quite straightforward to implement in the case of a simple environment (Favretto-Anrès and Rabau 1997). But for more complex configurations, for instance, setups with strongly curved interfaces generating wave focusing/defocusing, or setups prone to multiple diffractions, a further investigation is necessary. Therefore, the framework must be adapted accordingly, preferentially by following a crossvalidation approach between numerical tools and the laboratory setup (Pageot et al., 2017, Solymosi et al., 2018). Indeed, the comparison between the experimental and numerical results usually emphasizes the improvements of the experimental setup that must be made to increase the accuracy (and decrease the uncertainties) of the experiments, and at the same time, it points out the limitations of the numerical tools that must be tackled. In this paper, using such a cross-validation, we establish a hierarchical list of points to which particular attention should be paid to allow laboratory experiments to be very efficient tools in support of real data and numerical simulations for seismic exploration. Far from being exhaustive, this list may, however, be a useful starting point for similar studies combining laboratory experiments and numerical simulations. For the sake of illustration we rely on a much more complex case study that is also much more realistic from a geological viewpoint than the configurations considered in our previous works (Tantsereva et al., 2014a, Solymosi et al., 2018) or configurations considered in other works (e.g. Cooper et al., 2010, Stewart et al., 2012, Chang et al., 2017, Pageot et al., 2017), namely a complex-shaped salt body buried in sedimentary layers with curved surfaces. This choice is motivated by the fact that salt structures usually play a crucial role in hydrocarbon migration and entrapment, and as such receive special attention from exploration geophysicists. For many years various works have been published on (sub-)salt imaging, focusing on either the post-processing of already acquired datasets (e.g., Roberts et al., 2016), or on the acquisition techniques and geometries (e.g., Fleury, 2013). However, despite advances in migration algorithms and inversion methods, accurate (sub-)salt imaging is still an ongoing challenge for seismic exploration (Zhu et al., 2009, Jones and Davison, 2014, Liu et al., 2015). The main reason essentially stems from the particular properties of salt bodies. Indeed, salt often behaves and moves similarly to fluids at the geologic time scale, and salt bodies can, therefore, have various complex shapes, ranging from horizontal sheets to vertical "plums" with steep dips, or even mushroom or dome-shaped forms (e.g., Tari et al., 2003, Jackson and Hudec, 2017). Moreover, salt bodies usually have higher velocities than the typical surrounding sedimentary layers, while their density is generally close to that of the sediments, thus leading to a high reflectivity at the salt/sediments interfaces. As a consequence, imaging structures within and below the salt layers (also known as sub-salt imaging) is highly challenging, although inevitable for the detailed understanding of the complete geologic setup.

Motivated by the challenges related to salt bodies, we have built the WAVES reduced-scale 
model (scale ratio 1:20 000), closely representing both the geometry and the media properties of a realistic 3D geologic setup by using materials such as specific crystal and resins. The model represents a salt body buried in different sedimentary layers with curved surfaces, all of them on the top of a crystalline basement. The sedimentary layers have varying properties, including a zone with an inverted velocity profile where the velocity decreases with increasing depth. This model illustrates the current ability to design and manufacture realistic complex reduced-scale models of high interest for seismic exploration issues, using processes combining subtractive manufacturing and 3D printing. Zero-offset and offset reflection datasets have been then acquired for this reduced-scale model in a water tank, using a conventional pulse-echo technique and a broadband/broad-beam piezoelectric transducer and a hydrophone. To identify the points that need particular attention, we have compared these experimental datasets to numerical data obtained using a full-wave method, namely a spectralelement method (SEM) (Komatitsch and Vilotte 1998). To be effective, this cross-validation requires that the same input data (e.g., the geometry and the physical properties of the model, the characteristics of the sources and the receiver, and the acquisition design) have to be considered carefully in both the experiments and the numerical simulations. Finally, we have tested the validity of the whole framework (both the laboratory experiments and the numerical implementation) by applying a standard imaging technique of seismic exploration, namely Reverse-Time Migration (RTM) (Robein, 2010, Zhou et al., 2018) to the laboratory datasets.

The paper is organized as follows. The WAVES realistic geologic model presents the geometry and the properties of the reduced-scale model used for the laboratory experiments. Reduced-scale seismic modeling is devoted to the reduced-scale modeling, including the experimental setup and the data acquisition. In Numerical modeling the principles of the SEM used for obtaining numerical data similar to laboratory data are briefly reviewed. The numerical implementation of the input data similar to laboratory conditions is also discussed there. Comparisons between experimental and numerical data presents the comparisons between the laboratory data and the corresponding numerical results and discusses the possible origins of the observed misfits. More specifically, there, we provide a hierarchical list of points to which particular attention should be paid to allow laboratory experiments to be even more efficient tools to support real data and numerical simulations in seismic exploration. Finally, in the last section, RTM is applied successfully to the laboratory datasets, thus validating the whole framework and confirming the highly valuable potential of laboratory experiments for investigating specific seismic exploration issues.

\section{THE WAVES REALISTIC GEOLOGIC MODEL}

The real-life dimensions are scaled down to the laboratory scale by a factor of 1:20 000. Therefore, $1 \mathrm{~mm}$ at the laboratory scale corresponds to $20 \mathrm{~m}$ at seismic scale. The WAVES reducedscale model has dimensions of $400 \times 270 \times 95 \mathrm{~mm}^{3}$, which corresponds to $8 \times 5.4 \times 1.9 \mathrm{~km}^{3}$ at seismic scale. The model mimics a salt-body embedded in sedimentary layers (Figure 1). The manufacturing of the WAVES model was a challenging task due to both the choice of the relevant materials and to find an appropriate, precise and efficient manufacturing process. Salt is represented by crystal (glass enriched with lead monoxide) and the sediments are represented by resins (Table 1). The resins are all based on the same base material, but some of them are enriched with a mixture of aluminum and silicon dioxide powder to increase their density and their wavespeed. The aluminum layer on the bottom represents a typical crystalline basement, such as granite. The glass dome was manufactured with the help of a 3D-printed mold by La Fonderie de Verre, while the other parts were manufactured by VN Composites, who also assembled the whole physical model. The assembly started with the 
aluminum plate, on which the first resin layer was poured (layer \#4 in Figure 1). Then, it was constantly rotated in an oven where the temperature was gradually decreased over a few hours to avoid air bubbles forming in the resin, and also to ensure the homogeneous distribution of the Al$\mathrm{SiO}_{2}$ powder in the base resin. After solidifying, the top surface of the resin layer was carved out according to the curved surfaces described in the preliminary 3D plans. Finishing the fabrication of the first resin layer, the process continued with the next layer above (layer \#3 in Figure 1), and so on. Before adding the topmost resin layer (layer \#1 in Figure 1), the glass dome was placed inside. Its coupling with the surrounding layers has been ensured by a very thin layer of the base resin. The Pand S-wave velocity and attenuation values (expressed as Q-factors), obtained with transmission measurements through two material samples of different thickness, are close to constant within the frequency range of interest $(250-650 \mathrm{kHz})$. Each material is therefore considered to be homogeneous and isotropic for the frequency range of interest (Table 1). We consider glass and aluminum to be elastic, i.e. Qp and Qs are infinite.
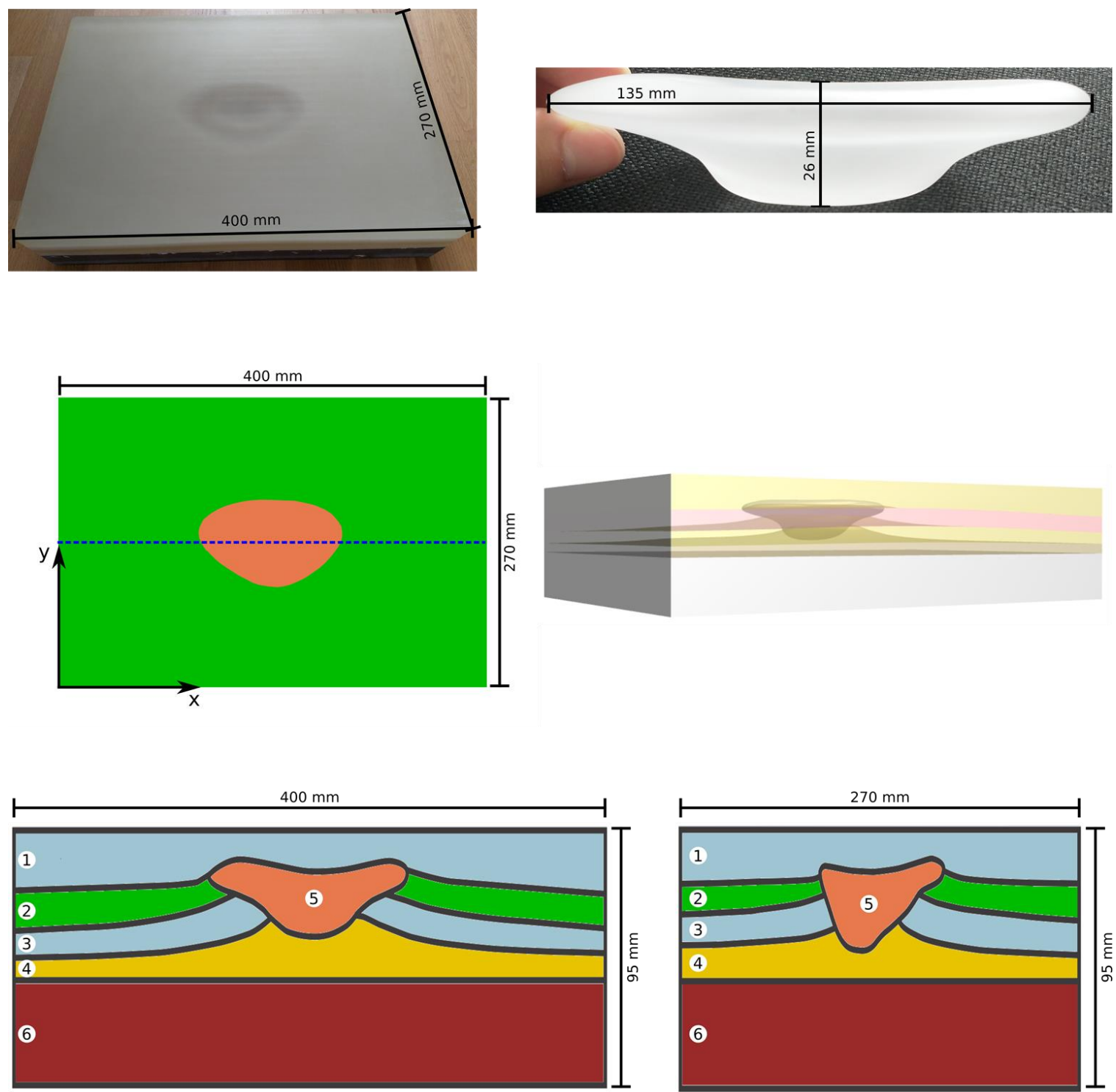

Figure 1. (a) The WAVES model after the assembly, (b) the glass dome placed inside, (c) top view of the WAVES model without the topmost layer, where the dashed blue line denotes the study line discussed in the paper, (d) illustration of the 3D geometry of the model, and (e and f) perpendicular vertical cross sections along the center lines of the model. The numbers denote the different materials whose properties are reported in Table 1. 


\begin{tabular}{|c|c|c|c|c|c|c|}
\hline Material & $\begin{array}{c}\text { Number in } \\
\text { Figure 1 }\end{array}$ & $\begin{array}{c}\text { Density } \\
\left(\mathbf{k g} / \mathbf{m}^{\mathbf{3}}\right)\end{array}$ & $\mathbf{V P}(\mathbf{m} / \mathbf{s})$ & $\mathbf{V s}(\mathbf{m} / \mathbf{s})$ & $\mathbf{Q P}_{\mathbf{P}}$ & $\mathbf{Q s}_{\mathbf{s}}$ \\
\hline resin A & 1,3 & $\begin{array}{c}1172 \pm 2 \\
( \pm 0.2 \%)\end{array}$ & $\begin{array}{c}2720 \pm 13 \\
( \pm 0.5 \%)\end{array}$ & $\begin{array}{c}1210 \pm 144 \\
( \pm 12 \%)\end{array}$ & $\begin{array}{c}25 \pm 1 \\
( \pm 4 \%)\end{array}$ & $\begin{array}{c}11 \pm 4 \\
( \pm 36 \%)\end{array}$ \\
\hline resin B & 2 & $\begin{array}{c}1680 \pm 10 \\
( \pm 0.6 \%)\end{array}$ & $\begin{array}{c}3090 \pm 16 \\
( \pm 0.5 \%)\end{array}$ & $\begin{array}{c}1577 \pm 25 \\
( \pm 1.6 \%)\end{array}$ & $\begin{array}{c}26 \pm 1 \\
( \pm 4 \%)\end{array}$ & $\begin{array}{c}18 \pm 3 \\
( \pm 17 \%)\end{array}$ \\
\hline resin C & 4 & $\begin{array}{c}1800 \pm 10 \\
( \pm 0.6 \%)\end{array}$ & $\begin{array}{c}3470 \pm 21 \\
( \pm 0.6 \%)\end{array}$ & $\begin{array}{c}1840 \pm 101 \\
( \pm 5 \%)\end{array}$ & $\begin{array}{c}53 \pm 1 \\
( \pm 2 \%)\end{array}$ & $\begin{array}{c}33 \pm 9 \\
( \pm 27 \%)\end{array}$ \\
\hline crystal glass & 5 & $\begin{array}{c}3623 \pm 10 \\
( \pm 0.3 \%)\end{array}$ & $\begin{array}{c}4480 \pm 43 \\
( \pm 1 \%)\end{array}$ & $\begin{array}{c}2845 \pm 464 \\
( \pm 16 \%)\end{array}$ & $\infty$ & $\infty$ \\
\hline aluminum & 6 & $\begin{array}{c}2710 \pm 4 \\
( \pm 0.2 \%)\end{array}$ & $\begin{array}{c}6441 \pm 87 \\
( \pm 1.4 \%)\end{array}$ & $\begin{array}{c}3573 \pm 544 \\
( \pm 15 \%)\end{array}$ & $\infty$ & $\infty$ \\
\hline
\end{tabular}

Table 1. Measured properties of the materials used in the WAVES model, together with the associated uncertainties for the frequency range of interest $(250-650 \mathrm{kHz})$.

\section{REDUCED-SCALE SEISMIC MODELING}

\section{Experimental setup}

Because the objective was to perform reduced-scale offshore seismic acquisitions, the WAVES model was immersed in a water tank before the measurements (Figure 2). The tank is equipped with a computer-controlled acquisition system that allows for the accurate positioning of the ultrasonic transducers. We emphasize here that, compared to the experimental setup described in Solymosi et al. (2018), the precision of the measurements has been significantly improved. The uncertainty of the transducer movements has been reduced to $\pm 5 \mu \mathrm{m}$ (i.e. $\pm 0.1 \mathrm{~m}$ at seismic scale), while the tilt angle of the source transducer can now be measured with a precision of $\pm 0.1^{\circ}$. Namely, optic rulers have been installed to provide a precise a posteriori control of the transducer movements, as well as a digital protractor to measure the tilt angle of the source transducer. It is estimated that, due to these modifications, the precision of the transducer movement and that of the source tilt angle have been improved by a factor of 100 and 10, respectively. However, determining with high accuracy the initial position of both the transducer and the hydrophone before the data acquisition is still a challenge. So far, this accuracy cannot be improved to better than $\pm 0.5 \mathrm{~mm}$, and this is definitely a point to further investigate and improve in future experiments. 

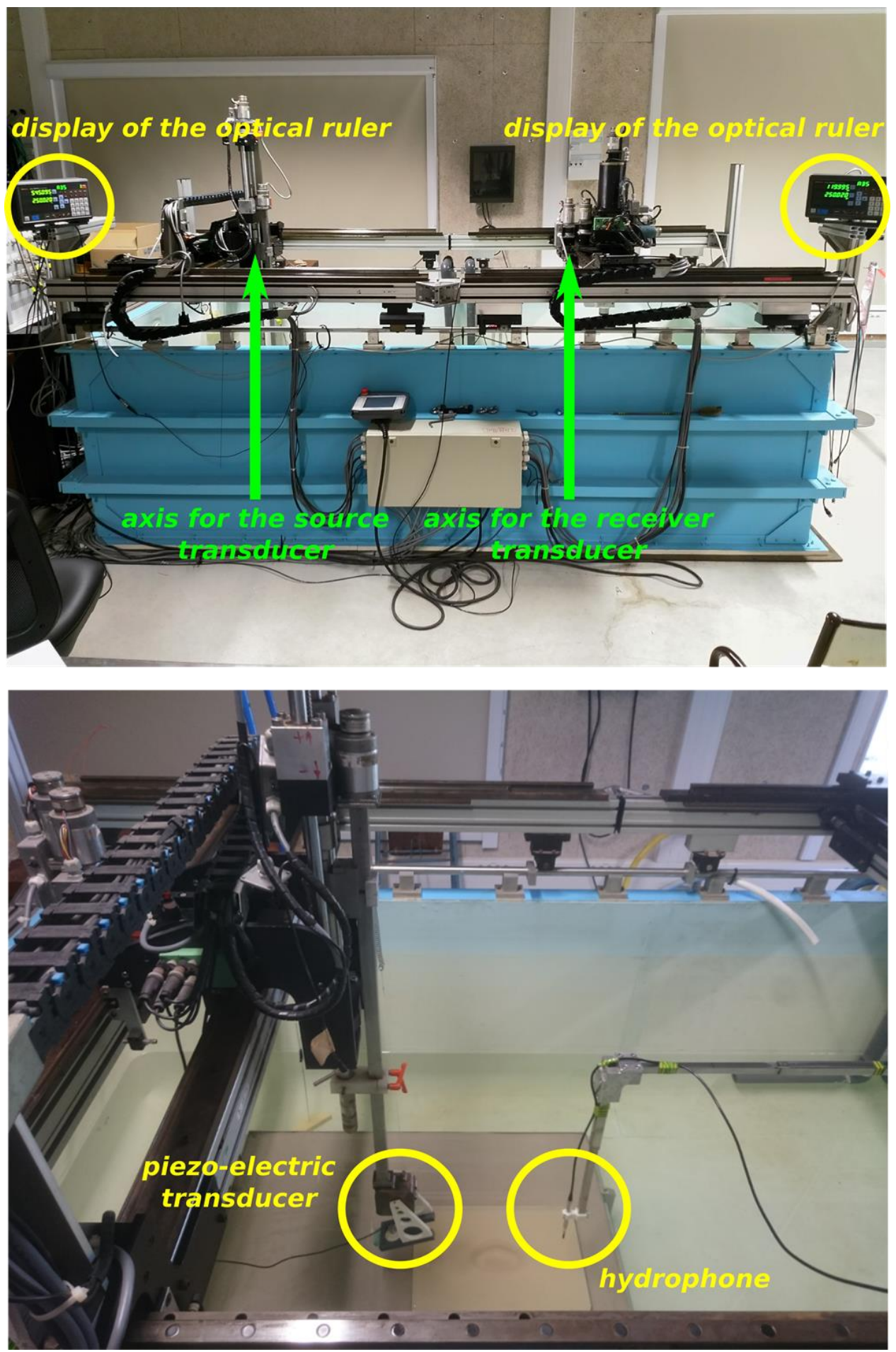

Figure 2. The water tank used for the experiments: (a) two axes support the two transducers; the movement of the source (four degrees of freedom) and that of the receiver (three degrees of freedom) are ensured by stepping-motors installed on the axes and controlled by a PC, whereas the recently installed optical rulers provide a posteriori control of the transducer movements and (b) the WAVES model placed inside the water tank.

As in Solymosi et al. (2018), we used a conventional pulse-echo technique to acquire reflection data in both zero-offset and offset configurations (Figure 2). Zero-offset data was acquired using a custom-made Imasonic $®$ transducer as both the source and the receiver. This transducer has 
a dominant frequency of $500 \mathrm{kHz}$ (i.e., $25 \mathrm{~Hz}$ at seismic scale) and a broad-beam radiation pattern. Because the width of the main lobe is $35^{\circ}$ at $-3 \mathrm{~dB}$, this transducer has a large area of illumination, leading to a complex 3D wavefield (Tantsereva et al., 2014a, 2014b, Solymosi et al., 2018). In the offset configuration, an omnidirectional Teledyne Reson ${ }^{\circledR}$ hydrophone was used as the receiver, which has a constant sensitivity for the frequency range of interest. For more details on the laboratory measurements, we refer the reader to Solymosi et al. (2018).

\section{Laboratory data sets}

For the sake of brevity, we focus on a study line, located above the center line in the x-direction (dashed line in Figure 1c, and along the section shown in Figure 1e). This acquisition line covers both rather simpler parts close to the sides where the geometry consists of close-to-horizontal layers, as well as complex parts in the center above the dome.

Figure 3 shows the laboratory zero-offset data set for the study line together with the interpretation of the main recorded events. The transducer was positioned $100.0 \pm 0.1 \mathrm{~mm}$ above the top surface of the model (i.e., $2000 \pm 2 \mathrm{~m}$ at seismic scale) and recorded events every $0.5 \mathrm{~mm}$ in the $\mathrm{x}$ direction. Event (a) represents reflections from the top surface of the model, i.e. from the top of the upper resin A layer. Event (b) represents mainly the reflections from the top of the glass dome, in particular for positions $120-270 \mathrm{~mm}$. For positions less than $120 \mathrm{~mm}$ and greater than $270 \mathrm{~mm}$ diffractions from the edges of the dome can also be observed. Events (c)-(f) are associated with the top surface of resin B, lower resin A, resin C, and aluminum, respectively. These events can be easily interpreted on the sides due to the relatively simple geometry. To the contrary, the closer we are to the center of the model, the harder it is to distinguish the same reflections due to the complex geometry. Due to the broad-beam radiation pattern of the source transducer and the curved top surface of the dome, we observe a constructive interference of reflections in the center, leading to focusing of the energy, between 165-200 $\mu$ s and positions 170-240 mm. Event ( $\mathrm{g}$ ) shows reflections from the bottom of the aluminum. The fact that reflections are recorded from the bottom of the aluminum shows that the imaging of the entire depth of the model is possible, even though the resin layers are highly attenuating (Table 1). Although events (f) and (g) represent reflections from two perfectly horizontal interfaces, they exhibit some undulations in Figure 3, which is indeed a velocity pull-up effect due to the time-domain visualization. This is the result of the varying velocity of the complex overburden of the aluminum, which then leads to different arrival times of the reflected zero-offset waves at different horizontal positions. 

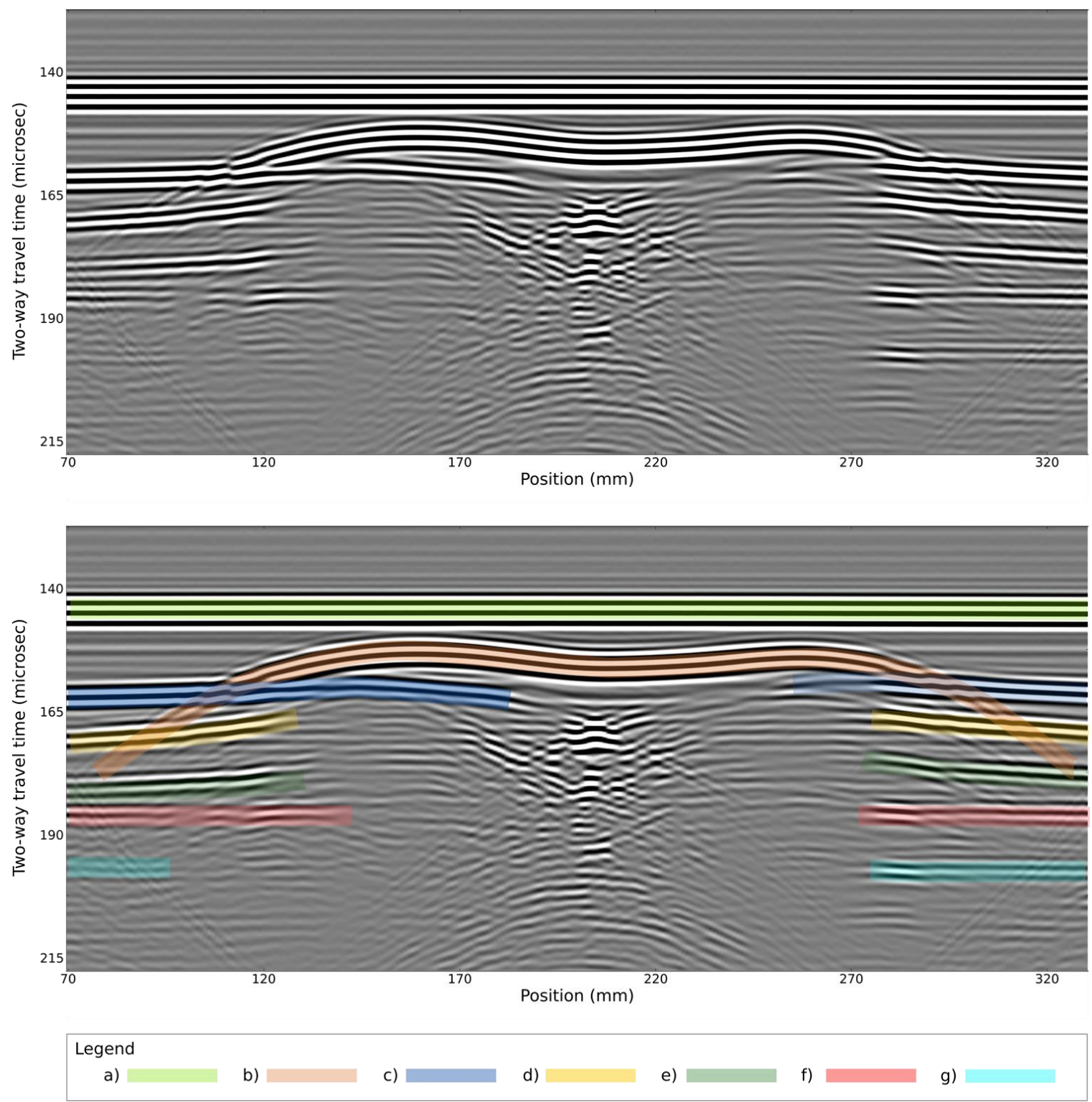

Figure 3. Cross section of the laboratory zero-offset data set along the study line (top) and interpretation (bottom). Annotated events: reflections from the (a) top of the upper resin A layer, (b) top of the glass dome, (c) top of the resin B layer, (d) top of the lower resin A layer, (e) top of the resin $\mathrm{C}$ layer, (f) top of the aluminum layer, and (g) bottom of the aluminum layer. The data were filtered between 250 and $650 \mathrm{kHz}$.

Figure 4 shows the laboratory offset data set for the study line, i.e. a common shot gather, together with the interpretation of the main events. The source was positioned at $y=390.17 \pm 0.50 \mathrm{~mm}$ and the tilt angle of the source transducer was $30.0 \pm 0.1^{\circ}$. Both the source and the receiver transducers were positioned $100.0 \pm 0.1 \mathrm{~mm}$ above the top surface of the model (i.e. $2000 \pm 2 \mathrm{~m}$ at seismic scale). The hydrophone was moved every $0.5 \mathrm{~mm}$ in the $\mathrm{x}$-direction to record events. The interpretation of the measured data set for the WAVES model without post-processing is challenging due to the complex multi-layered geometry. Nevertheless, event (a) can be unambiguously attributed to the direct arrival from the source, and event (b) to the reflections from the top surface of the model, i.e. from the top of the upper resin A layer. To go further in the interpretation, the laboratory data must be post-processed, most typically with migration algorithms. We show an example of this below. 

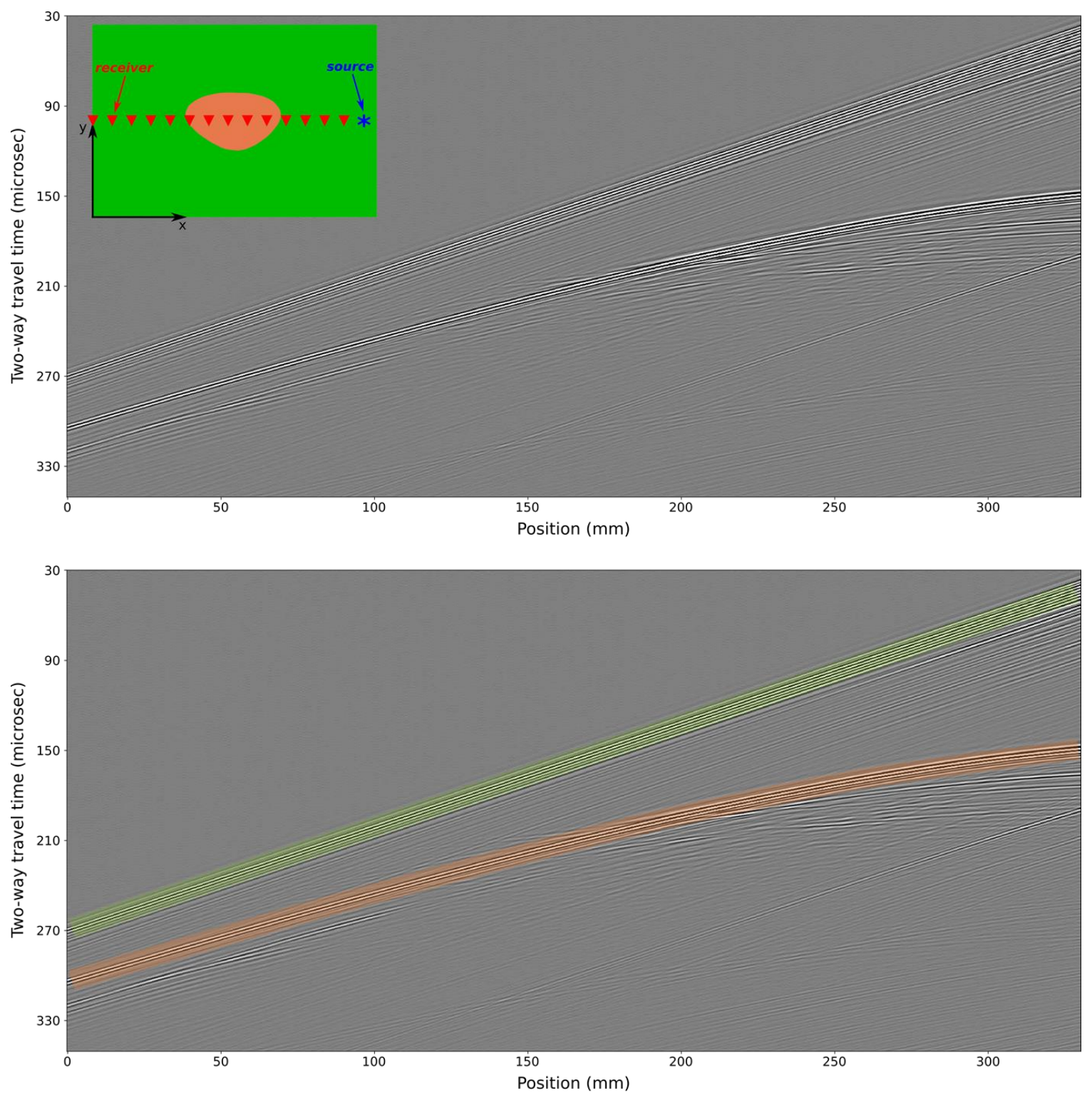

Legend

a)

b)

Figure 4. Cross section of the laboratory offset data set along the study line (i.e., common-shot gather) (top), and interpretation (bottom). Annotated events: (a) direct arrival and (b) reflection from the top of the upper resin A layer. The data were filtered between 250 and $650 \mathrm{kHz}$.

\section{NUMERICAL MODELING}

\section{Brief description of the numerical method}

We resorted to the spectral-element method (SEM) (e.g., Komatitsch and Vilotte, 1998, Fichtner, 2010, Peter et al., 2011) for the numerical simulations. Specifically, we used the SPECFEM software package (Komatitsch and Vilotte, 1998) with the explicit second-order Newmark time scheme (Hughes, 1987). The numerical modeling is conducted in the same way as reported in Solymosi et al. (2018), including the implementation of the viscoelasticity and the real transducer characteristics. The only difference lies in the implementation of the model geometry. Because the 
WAVES model has a complex 3D multi-layered geometry, obtaining a non-structured hexahedral mesh is extremely difficult (e.g., Shepherd and Johnson, 2008, Staten et al., 2010). The main difficulty is not only to correctly mesh any domain of the model with hexahedral elements only, but also to have a conform mesh on the boundaries between any two domains. Indeed, in a conform mesh all nodes which are on the boundary of two domains are shared by elements on both sides, and all elements on the boundaries must be connected to elements in the other domain by nodes. Although several opensource and commercial meshing software have been tested, currently they are all unable to tackle this task for the WAVES model. Because a conformal mesh is necessary for the numerical simulations, we had to choose a structured grid to numerically implement the model geometry. Considering the minimum velocity of the model - namely, $1210 \mathrm{~m} / \mathrm{s}$ for the S-waves in resin A - and the maximum target frequency $(650 \mathrm{kHz})$, we used a grid with an equidistant grid spacing of $1.6 \mathrm{~mm}$ in each spatial direction. Because five Gauss-Lobatto-Legendre (GLL) points per element are used during the simulations (Solymosi et al., 2018), the grid spacing provides at least 7.1 and 5.8 GLL points per the shortest wavelength for P- and S-waves, respectively. This spatial discretization yields approximately 5.6 million elements, considering the water column above the model as well. We used Intel Xeon Sandy Bridge EP (E5-2690) processors for the simulations. The computational cost was 1500 core hours to simulate $350 \mu$ s of wave propagation (corresponding to $7 \mathrm{~s}$ at seismic scale).

\section{Numerical calibration of the material properties for a multi-layered model}

Because the ultrasonic characterization of the material samples yields a range of possible values for the measured properties due to the measurement uncertainties, an initial calibration is necessary to find the relevant values to be used for the simulations. The calibration consists of a zerooffset laboratory measurement, followed by an iterative fitting of the corresponding synthetic trace. This iterative fitting consists of the adjustment of the material parameters of each layer such that the simulation yields the closest possible fit with the reference laboratory trace. Compared to the Marseille-Benchie model (Solymosi et al., 2018), where the properties of only one material had to be fitted, the WAVES model is more challenging due to its multi-layered geometry. Our strategy is simple: at first, the properties of the topmost layer are calibrated, then, considering these calibrated values, the properties of the second layer are calibrated, and so on. We chose a reference point $100.0 \pm 0.1 \mathrm{~mm}$ above the top surface of the model as close to the sides of the model as possible where the geometry is close to a 2D layer-cake geometry, but avoiding diffractions effects from the model edges (Figure 5). The calibration thus consists of two phases: first, the properties of each layer except those of the glass dome are calibrated successively using the reference point, then, a second trace is used in the center of the model to also fit the properties of the glass (Figure 5).

Figure 6a shows the comparison of the reference laboratory trace with the simulated trace, using the measured material properties reported in Table 1. Although the arrival time, amplitude and phase of the simulation are correct for the first event (reflection from the top of the upper resin A layer), the later arrivals have extremely low amplitudes compared to the laboratory measurement. By successively adjusting the material parameters of the different layers, we can finally obtain an excellent fit between the laboratory and synthetic traces, as shown in Figure 6b, from a quantitative (through correlation coefficients) points of view. Table 2 reports the adjusted material properties and the change in terms of percentages compared to the measured material properties listed in Table 1. At this point, we note that the differences between the adjusted and the measured values of the Q-factors appears to be significant, whereas the differences in the velocity values remain small. Moreover, even though the same resin A is used twice in the model, we obtained different material properties for the 
upper and lower layers, especially for the attenuation parameters. Finally, using the calibrated material properties of Table 2, we did an additional control measurement and simulation in the same horizontal position, but one centimeter closer to the surface of the model (i.e. at $90.0 \pm 0.1 \mathrm{~mm}$ above the top surface of the model). Changing only the height of the transducer results in a slightly different fit between laboratory and synthetic traces (Figure 6). It can be explained by the fact that the width of the illuminated zone of the model changes with the transducer height. Here, we can clearly see the importance of the illuminated zone on the measurements, and on the calibration as well, in particular for a multi-layered medium. Thus, the adjusted material parameters should be considered as rather apparent or effective parameters than intrinsic parameters of the materials. We note that the accurate and reliable estimation of the intrinsic material attenuation is still a challenging issue both for the laboratory and the field experiments. Discussions about the relevancy of both the (classical) experimental procedure used here to evaluate intrinsic attenuation and the consideration of constant Q-factors in numerical models (as usually considered in seismic/seismological applications) are beyond the scope of this paper.

\begin{tabular}{|c|c|c|c|c|c|c|}
\hline Layer & $\begin{array}{c}\text { Number in } \\
\text { Figure } 1\end{array}$ & $\begin{array}{l}\text { Density } \\
\left(\mathrm{kg} / \mathbf{m}^{3}\right)\end{array}$ & $V_{P}(\mathbf{m} / \mathbf{s})$ & $\mathbf{V}_{\mathrm{S}}(\mathrm{m} / \mathbf{s})$ & $Q_{P}$ & Qs \\
\hline $\begin{array}{l}\text { upper } \\
\text { resin A }\end{array}$ & 1 & $\begin{array}{l}1172 \\
(0 \%)\end{array}$ & $\begin{array}{l}2549 \\
(-6 \%)\end{array}$ & $\begin{array}{l}1210 \\
(0 \%)\end{array}$ & $\begin{array}{c}123.8 \\
(376 \%)\end{array}$ & $\begin{array}{c}88 \\
(389 \%)\end{array}$ \\
\hline resin B & 2 & $\begin{array}{l}1680 \\
(0 \%)\end{array}$ & $\begin{array}{l}3213 \\
(4 \%)\end{array}$ & $\begin{array}{l}1577 \\
(0 \%)\end{array}$ & $\begin{array}{l}27.3 \\
(5 \%)\end{array}$ & $\begin{array}{c}23 \\
(28 \%)\end{array}$ \\
\hline $\begin{array}{l}\text { lower } \\
\text { resin A }\end{array}$ & 3 & $\begin{array}{l}1172 \\
(0 \%)\end{array}$ & $\begin{array}{l}2560 \\
(-6 \%)\end{array}$ & $\begin{array}{l}1210 \\
(0 \%)\end{array}$ & $\begin{array}{c}41.7 \\
(60 \%)\end{array}$ & $\begin{array}{c}30 \\
(67 \%)\end{array}$ \\
\hline resin $\mathrm{C}$ & 4 & $\begin{array}{l}1800 \\
(0 \%)\end{array}$ & $\begin{array}{c}3050 \\
(-12 \%)\end{array}$ & $\begin{array}{l}1840 \\
(0 \%)\end{array}$ & $\begin{array}{c}30 \\
(-43 \%)\end{array}$ & $\begin{array}{c}26 \\
(-21 \%)\end{array}$ \\
\hline aluminum & 6 & $\begin{array}{l}2710 \\
(0 \%)\end{array}$ & $\begin{array}{c}6491 \\
(<1 \%)\end{array}$ & $\begin{array}{l}3573 \\
(0 \%)\end{array}$ & $\begin{array}{c}\infty \\
(0 \%)\end{array}$ & $\begin{array}{c}\infty \\
(0 \%)\end{array}$ \\
\hline
\end{tabular}

Table 2. The calibrated material properties for the resins and the aluminum. The percentages show the differences compared to the measured values listed in Table 1. 


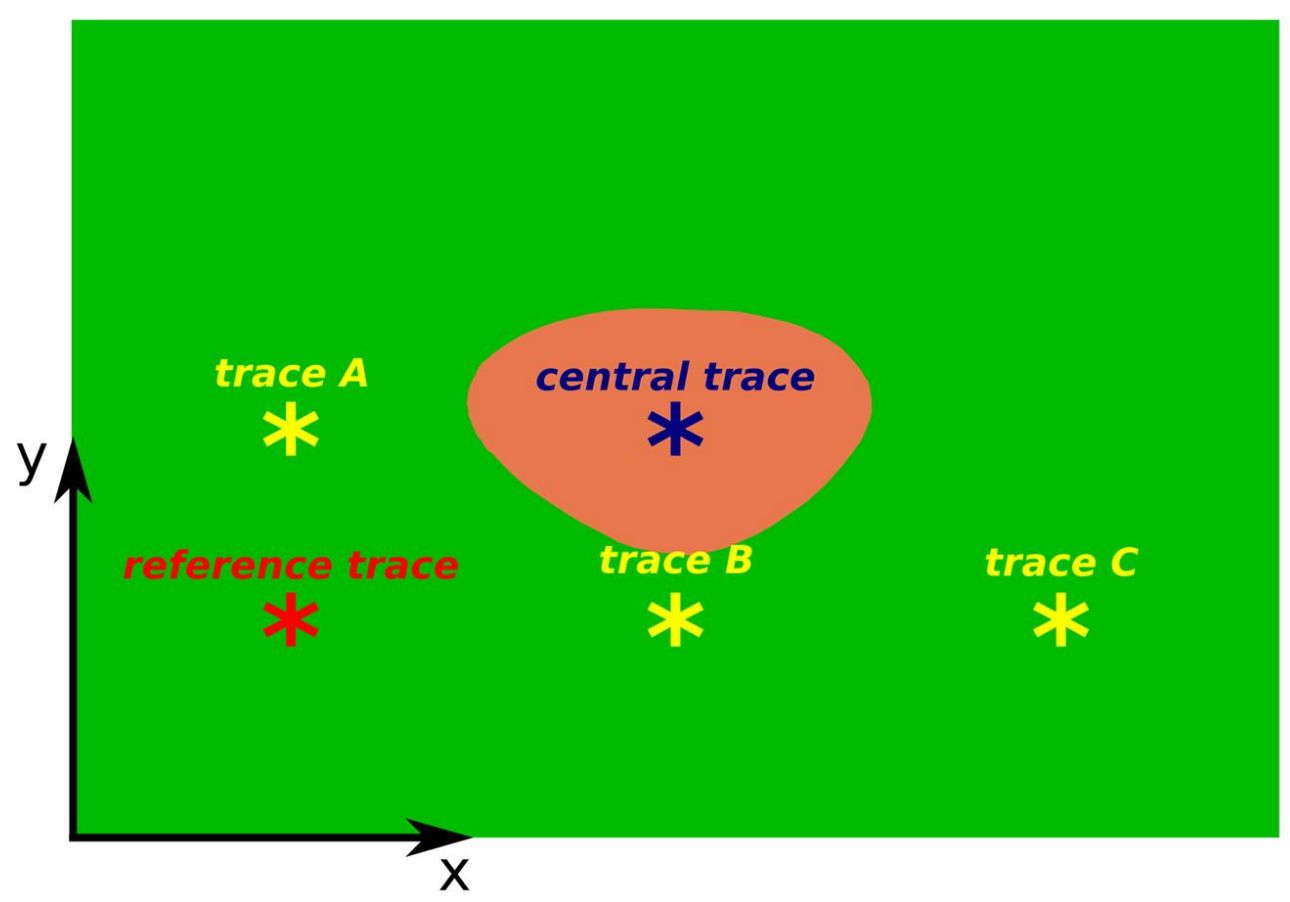

Figure 5. Location of the reference trace (red asterisk) and of the second trace used to calibrate the properties of the glass (blue asterisk). The yellow asterisks denote the position of the zero-offset examples showcased below.

a)

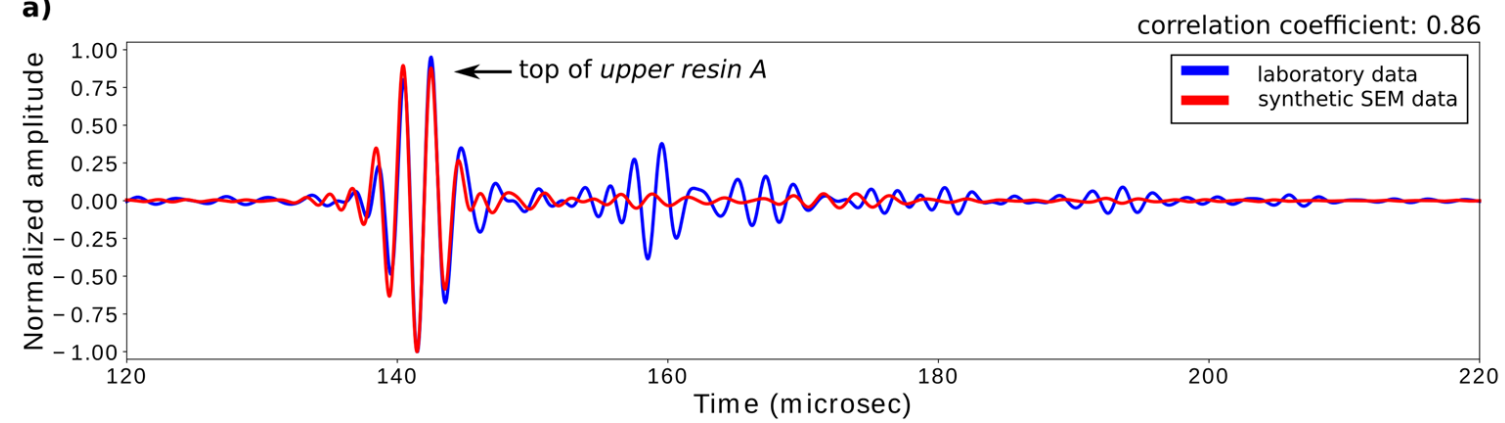

b)

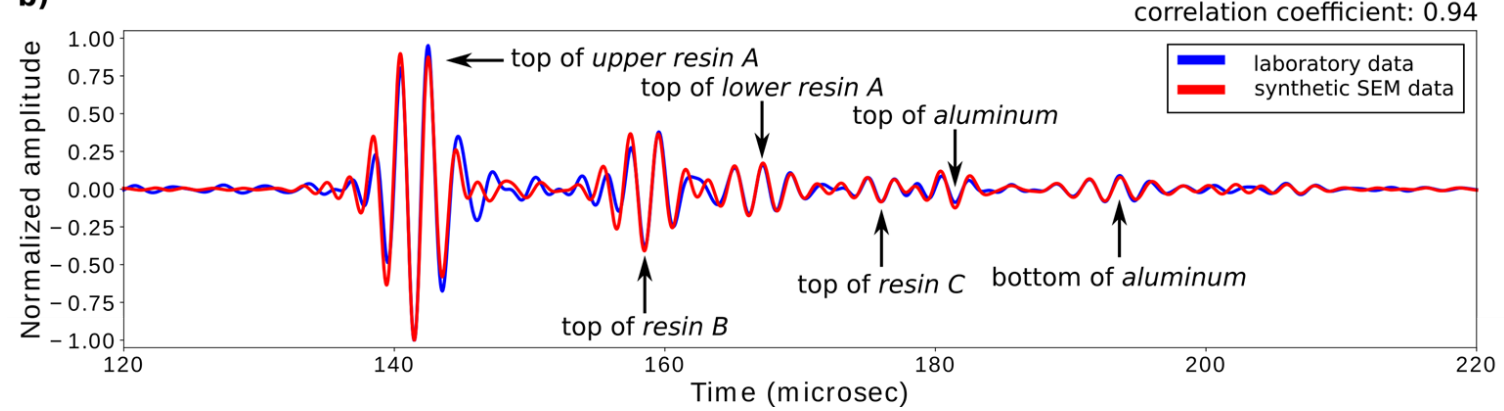

Figure 6. Comparison of the reference laboratory trace (blue) and the corresponding synthetic trace (red) obtained using: a) the measured material properties reported in Table 1, b) the adjusted material properties reported in Table 2. 


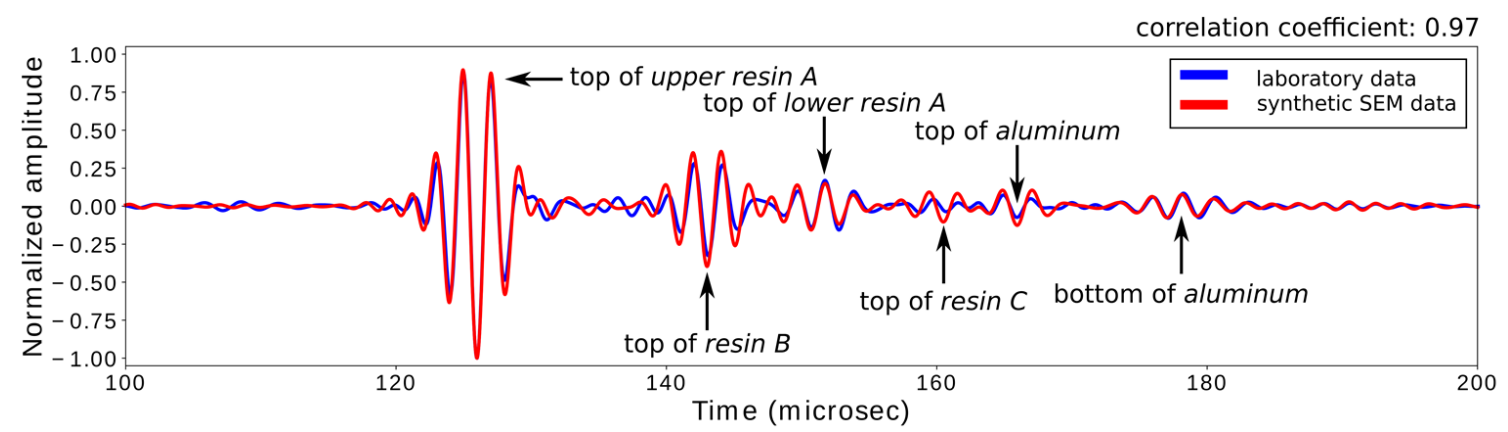

Figure 7. Comparison of the laboratory trace (blue) recorded at the same horizontal position as the reference trace (Figure 6), but one centimeter closer to the model, and the corresponding synthetic trace (red) obtained using the adjusted material properties reported in Table 2.

After calibrating the material properties for the resins and the aluminum, a second trace was used in the center of the model to adjust the properties of the glass (blue asterisk in Figure 5). Figure 8a shows the comparison of the laboratory trace with the synthetic results, using the adjusted material properties listed in Table 2. We can see that the two traces fit each other very well for the reflection from the top of the upper resin A layer. Figure $8 \mathrm{~b}$ shows the same comparison, but this time the material properties of the glass dome are also calibrated (Table 3). We can clearly see a good fit between the laboratory and synthetic traces for the reflection from the bottom of the glass dome too, as well as for other events with later arrival times. The reflection from the top of the glass dome shows a good, but not a perfect fit, as the amplitude is overestimated compared to the laboratory measurement. To decrease this misfit, the properties of upper resin A should have been readjusted. In other words, it would have required different properties of the same layer at different locations, meaning that the materials would have been heterogeneous. However, this heterogeneity was not confirmed by our measurements for the material samples, also including X-ray tomography. This misfit may be due to the effect of the illuminated zone, that also depends on the model geometry (especially in this case when the top surface of the dome is curved), and hence to mild focusing/defocusing effects.
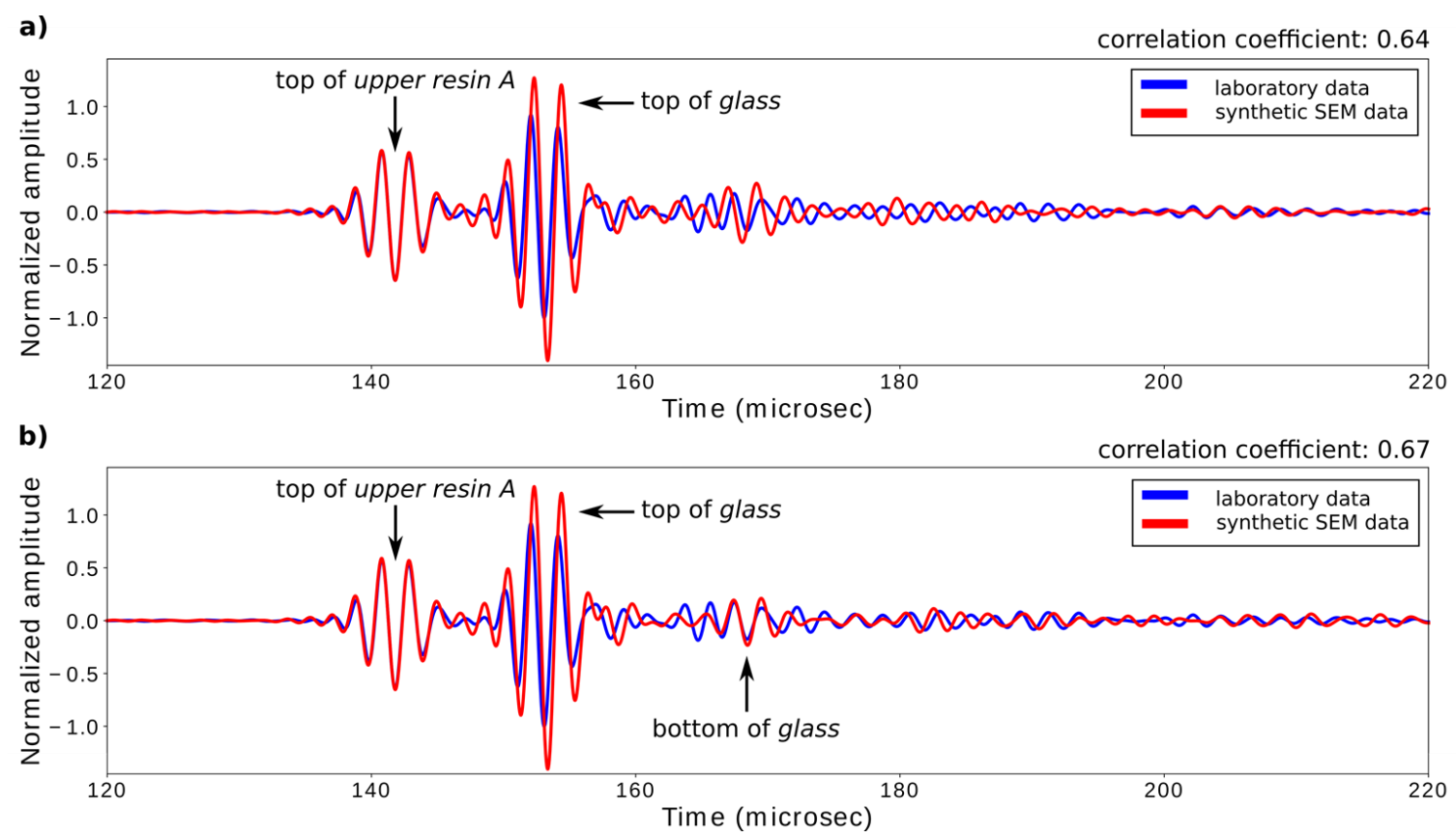

Figure 8. Comparison of the laboratory trace (blue) recorded in the center of the model and the 
corresponding synthetic trace (red) obtained using: a) the adjusted material properties listed in Table 2, b) the adjusted material properties reported in Tables 2 and 3.

\begin{tabular}{|c|c|c|c|c|c|c|}
\hline Layer & $\begin{array}{c}\text { Number in } \\
\text { Figure 1 }\end{array}$ & $\begin{array}{c}\text { Density } \\
\left(\mathbf{k g} / \mathbf{m}^{\mathbf{3}}\right)\end{array}$ & $\mathbf{V P ~}_{\mathbf{P}}(\mathbf{m} / \mathbf{s})$ & $\mathbf{V S ~}_{\mathbf{~}}(\mathbf{m} / \mathbf{s})$ & QP & Qs \\
\hline glass & 5 & $\begin{array}{c}3623 \\
(0 \%)\end{array}$ & $\begin{array}{c}4325 \\
(-4 \%)\end{array}$ & $\begin{array}{c}2845 \\
(0 \%)\end{array}$ & $\begin{array}{c}\infty \\
(0 \%)\end{array}$ & $\begin{array}{c}\infty \\
(0 \%)\end{array}$ \\
\hline
\end{tabular}

Table 3. The calibrated material properties for the glass. The percentages show the differences compared to the measured values reported in Table 2.

\section{COMPARISONS BETWEEN EXPERIMENTAL AND NUMERICAL DATA}

\section{Comparison of zero-offset data}

In addition to the reference and the central traces, we consider here more specifically three traces of the laboratory zero-offset data set, acquired at locations denoted with the yellow asterisks in Figure 5. These traces are located at different parts of the model to investigate the fit between the measurements and the simulations, using the calibrated material properties (Tables 2 and 3) at different positions.

Trace A is located $130 \mathrm{~mm}$ (i.e., $2.6 \mathrm{~km}$ at seismic scale) far from the reference trace in the $\mathrm{y}$ direction (Figure 5). The comparison of the laboratory measurement with the numerical result shows a general good fit for both the arrival times and the amplitudes for most of the events (Figure 9a), as highlighted by the high value (0.93) of the correlation coefficient of the two traces. In particular, the first three reflections (from the top of upper resin A, resin B and lower resin A layers, respectively) exhibit an almost perfect fit in terms of arrival time and amplitude, whereas the reflection from the top of resin $\mathrm{C}$ shows only some minor amplitude and arrival time misfits. On the contrary, the reflections from the top and bottom of the aluminum layer reveal a significant misfit in the arrival times.

Trace B is located $130 \mathrm{~mm}$ (i.e., $2.6 \mathrm{~km}$ at seismic scale) far from the reference trace in the $\mathrm{x}$ direction (Figure 5). Figure 9b shows a good fit between the measurements and the simulations for the reflection from the top of the upper resin A layer, except for the tail of the event which exhibits some minor amplitude misfits. Although some amplitude misfits can be observed, the reflections from the top of resin B and lower resin A layers are well restored by the simulations. On the contrary, the later arrivals of trace $\mathrm{B}$ (corresponding to reflections from the top of the resin $\mathrm{C}$ layer and to reflections from the top and bottom of the aluminum layer) show an almost perfect fit in both the arrival times and the amplitudes. Despite the occasional amplitude misfits, trace B has a high correlation coefficient (0.87).

Trace $\mathrm{C}$ is located $260 \mathrm{~mm}$ (i.e., $5.2 \mathrm{~km}$ at seismic scale) far from the reference trace in the $\mathrm{x}$ direction (Figure 5). Figure 9c shows the comparison between the measurement and the simulation, revealing a generally good fit. The fit is especially good for the reflections from the top of upper resin $\mathrm{A}$ and resin B layers. The reflections from the top of lower resin A and resin $\mathrm{C}$ layers, and from the bottom of the aluminum layer show some small amplitude and arrival time misfits. On the contrary, 
the arrival time misfit for the reflection from the top of the aluminum layer is significant. We also note the arrival recorded after the reflection from the top of the upper resin A layer, around $148 \mu$ s. This event is pronounced for the measurement, while not reconstructed by the simulation. This arrival is probably related to tiny air-bubbles which accumulated on the surface of the physical model during the measurements, which can take even several days for the entire data set. Nevertheless, trace $\mathrm{C}$ has a quite high correlation coefficient (0.88).

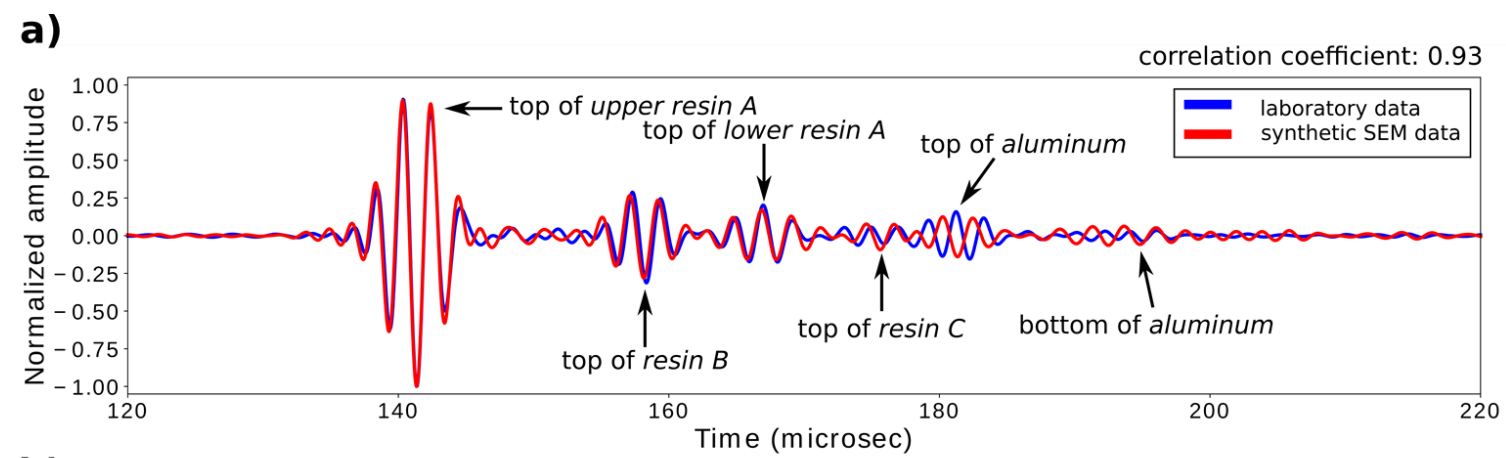

\section{b)}

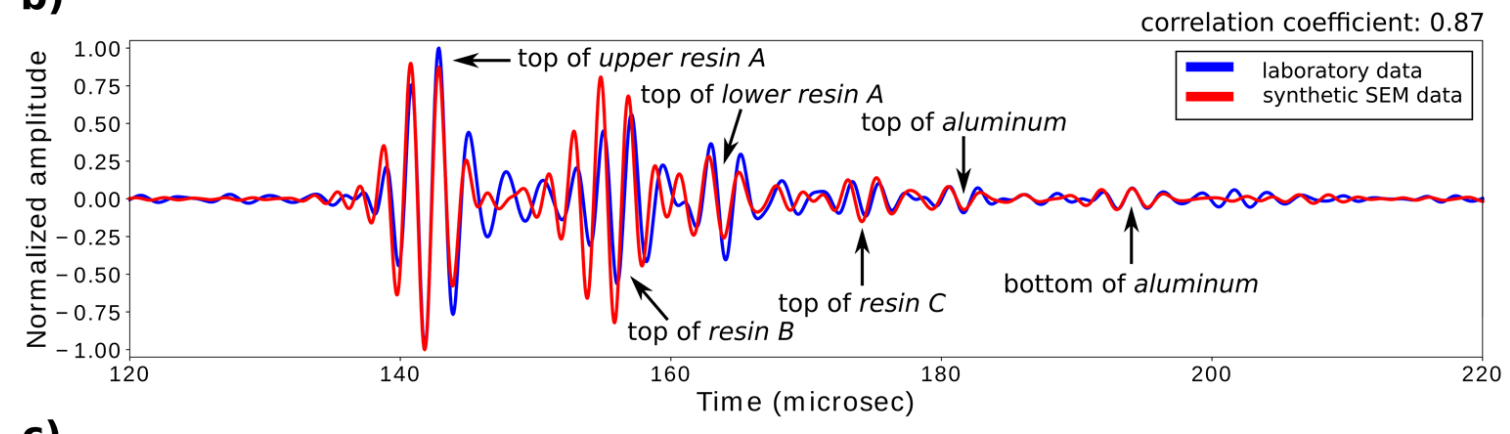

c)

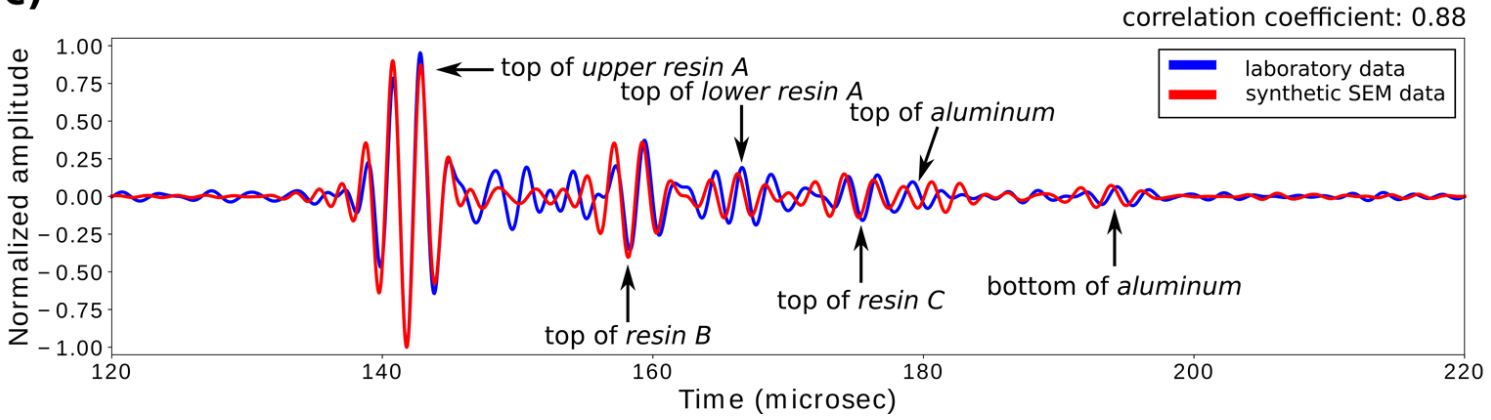

Figure 9. Comparison of zero-offset laboratory traces with the corresponding synthetic SEM results: a) trace $A, b)$ trace $B, c)$ trace $C$.

\section{Comparison of offset data}

Here, we consider more specifically two sets of offset traces, corresponding to two different source positions. All the sources and receivers were positioned in the center line of the model in the $\mathrm{y}$-direction $(\mathrm{y}=135.0 \pm 0.5 \mathrm{~mm})$. The two source positions are denoted with red and blue asterisks in Figure 10, respectively. The positions of the receivers are denoted with triangles of the corresponding color. The tilt angle of the source transducer for all the traces was $30.0 \pm 0.1^{\circ}$. 


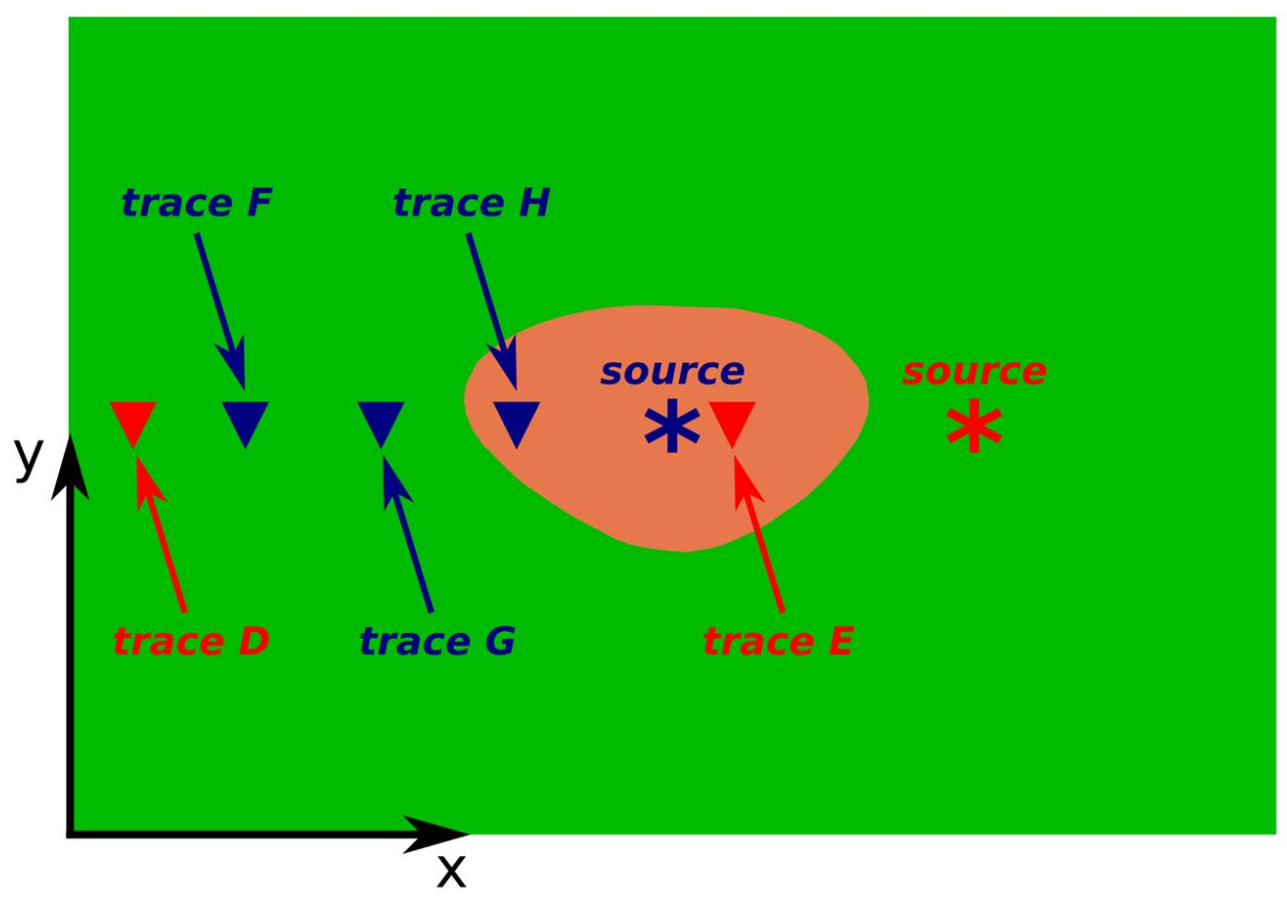

Figure 10. Location of the sources (asterisk) and receivers (triangles) associated to the two showcased offset experiments. The two data sets are denoted with red and blue colors, respectively.

\section{First set of offset traces}

The source was positioned at $\mathbf{x}=300.0 \pm 0.5 \mathrm{~mm}$ for traces D and E (Figure 10). Trace D corresponds to an offset of $280.0 \pm 0.5 \mathrm{~mm}$ (i.e., $5.60 \pm 0.01 \mathrm{~km}$ at seismic scale). Figure 11a shows the comparison of the laboratory measurement with the synthetic result. The reflection from the top of the upper resin A layer shows a good fit between the two traces, in terms of both the arrival time and the amplitude. Although the arrival time of the reflection from the top of the glass dome is correct for the synthetic trace, the simulated amplitude is somewhat lower compared to the measurement. The later arrivals, which propagated through the glass dome, cannot be easily interpreted one-by-one, because of complex wave paths. Although the arrival time and phase of these events are correct, they show a varying amplitude misfit. A correlation coefficient of 0.84 , however, suggests a generally good fit between the measurement and the simulation.

Trace $\mathrm{E}$ was recorded at an offset of $80.0 \pm 0.5 \mathrm{~mm}$ (i.e., $1.60 \pm 0.01 \mathrm{~km}$ at seismic scale). The comparison of the measured and simulated traces in Figure 11b shows a good fit for the reflection from the top of the upper resin A layer, similar to trace D. Following that, there are two arrivals corresponding to reflections from the top of the glass dome. This is due to the broad-beam radiation pattern of the source in combination with the curved top surface of the dome. These arrivals show some arrival time and amplitude misfits. The later arrivals, corresponding to waves propagating through the glass dome, show a similar pattern as for trace D. Namely, the arrival time of these events are mostly correct with some amplitude misfits. The correlation coefficient is 0.81 , partly due to the erroneously low simulated amplitude of the later arrivals (e.g., at $180 \mu \mathrm{s}$ ). 


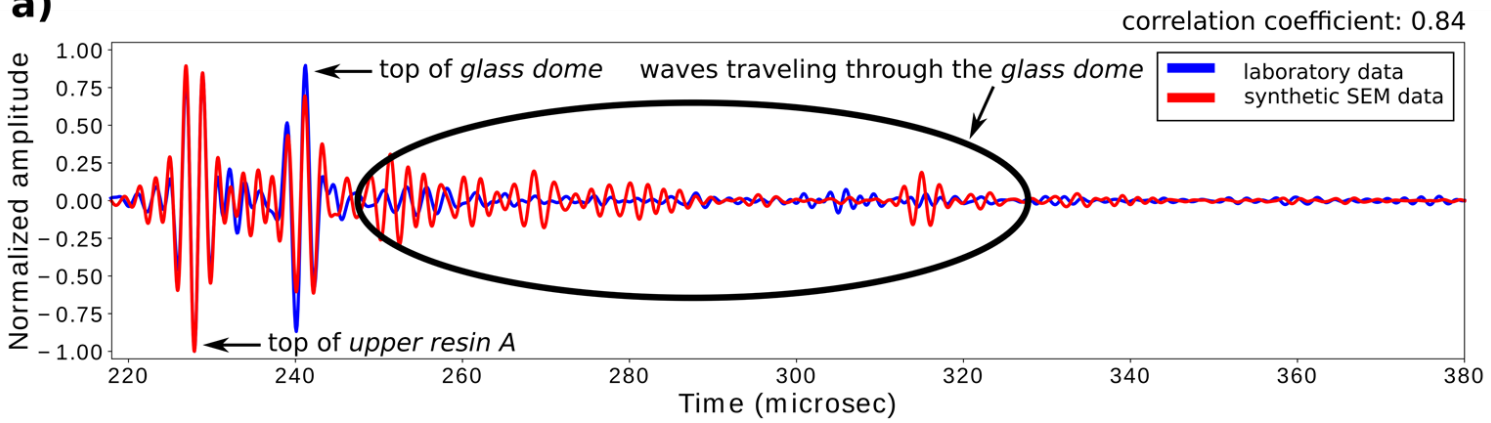

b)

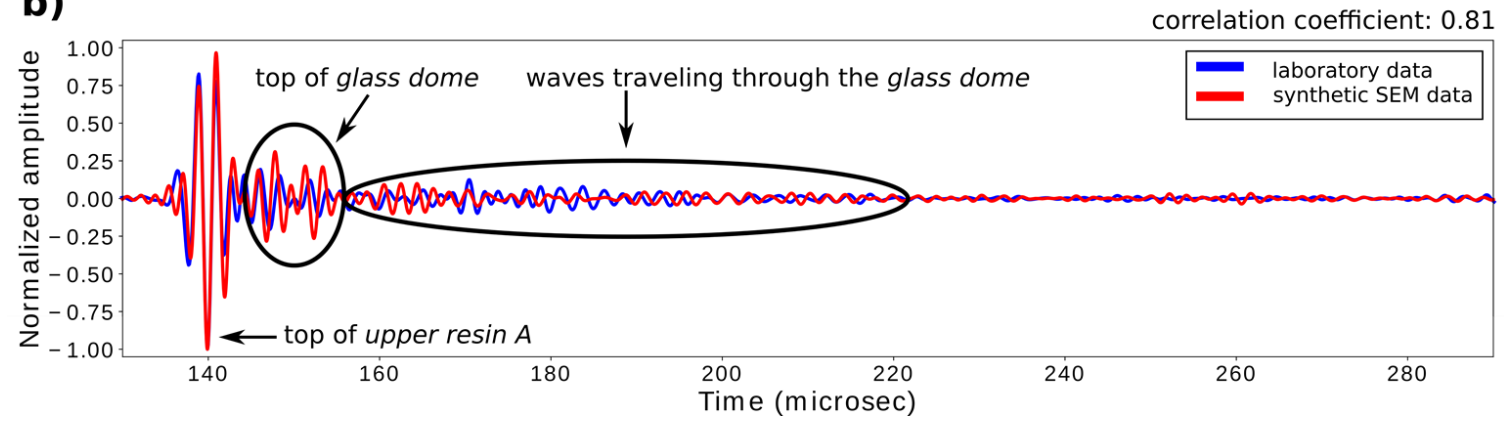

Figure 11. Comparison of offset laboratory traces with the corresponding synthetic SEM results: a) trace $\mathrm{D}, \mathrm{b})$ trace $\mathrm{E}$.

\section{Second set of offset traces}

For the second set of offset traces, the source was positioned at $\mathbf{x}=200.0 \pm 0.5 \mathrm{~mm}$ (Figure 10). Trace F corresponds to an offset of $140.0 \pm 0.5 \mathrm{~mm}$ (i.e., $2.80 \pm 0.01 \mathrm{~km}$ at seismic scale). The reflection from the top of the upper resin A layer has the correct arrival time and amplitude, but the phase of the early and late parts of this event is somewhat distorted, compared to the laboratory trace (Figure 12a). The reflection from the top of the glass dome shows mainly some amplitude misfit. The simulated arrivals of the waves traveling through the glass dome show an erroneously low amplitude in general, which explains the lower correlation coefficient of 0.78 compared with traces D and E above.

Trace $\mathrm{G}$ (Figure 12b) corresponds to an offset of $90.0 \pm 0.5 \mathrm{~mm}$ (i.e., $1.80 \pm 0.01 \mathrm{~km}$ at seismic scale). The reflection from the top of the upper resin A layer exhibits a good fit in time and amplitude. The early and late parts of this event have a somewhat superior fit in phase than for trace F. The two reflections from the top of the glass dome are of excellent fit in both arrival times and amplitudes. However, the later arrivals, corresponding to waves propagating through the dome, again reveal an erroneously low amplitude for the synthetic trace. The correlation coefficient is 0.81 , and this higher value compared with trace $\mathrm{F}$ is due to the better fit at earlier times.

Trace H (Figure 12c) corresponds to an offset of 50.0 $\pm 0.5 \mathrm{~mm}$ (i.e., $1.00 \pm 0.01 \mathrm{~km}$ at seismic scale). This near-offset trace shows an excellent fit in arrival times and amplitudes for the reflections from both the top of the upper resin A layer and the glass dome. For this trace, even the beginning of the complex arrivals related to the rays propagating through the glass dome exhibits a good fit between the simulation and the measurement. Indeed, the arrival at around $150 \mu$ s has both the good amplitude and arrival time with a minor phase misfit. The later parts of these arrivals also show a good fit in time. Similar to the previous traces, the simulated amplitude of these events after $180 \mu \mathrm{s}$ is generally too low, explaining the lower correlation coefficient of 0.78 . 
a)

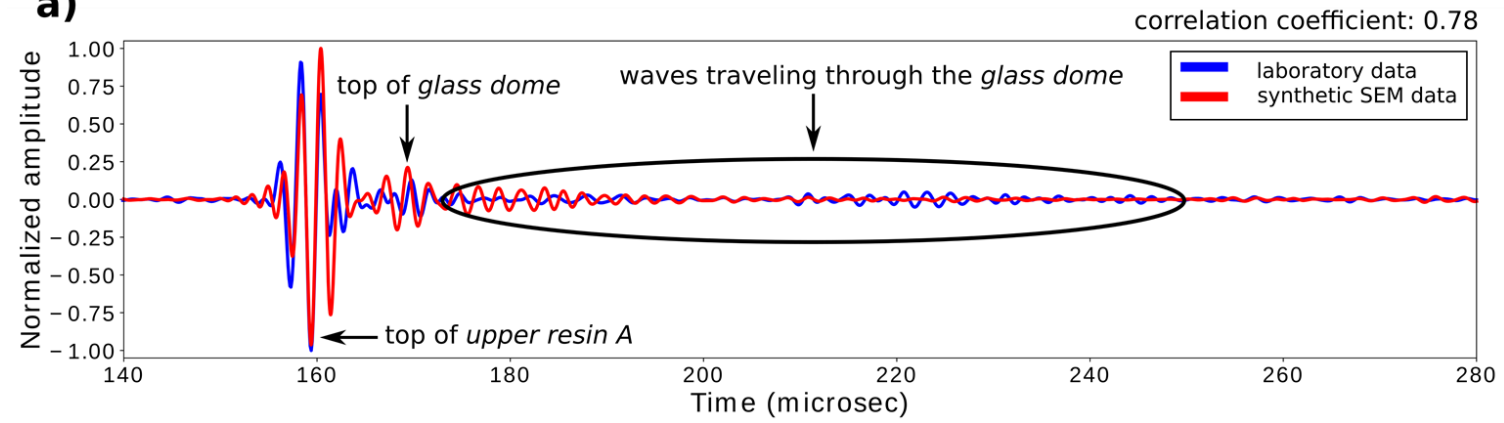

b)

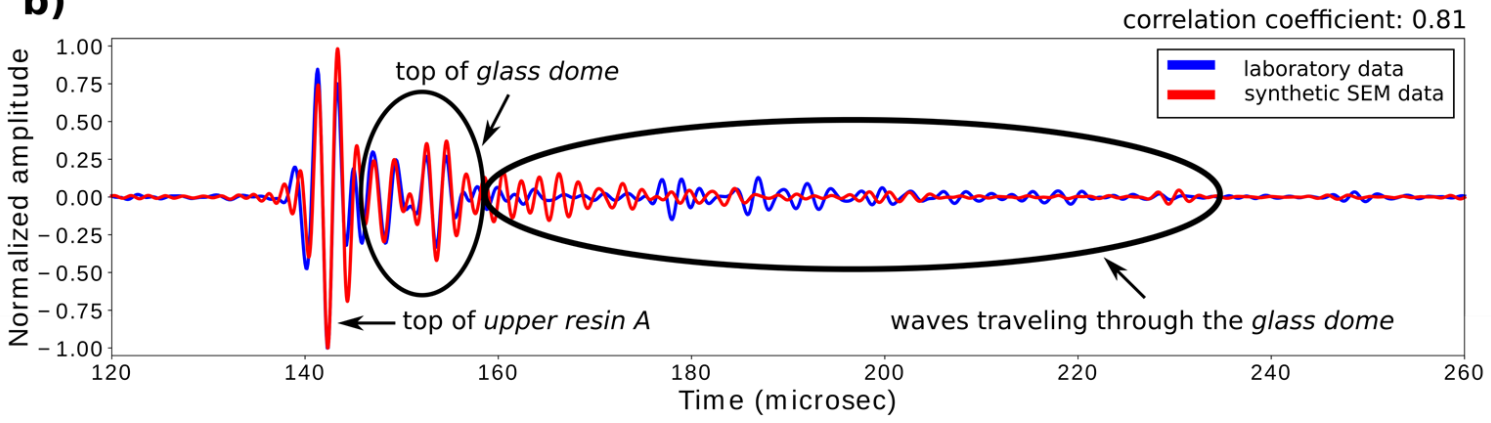

c)

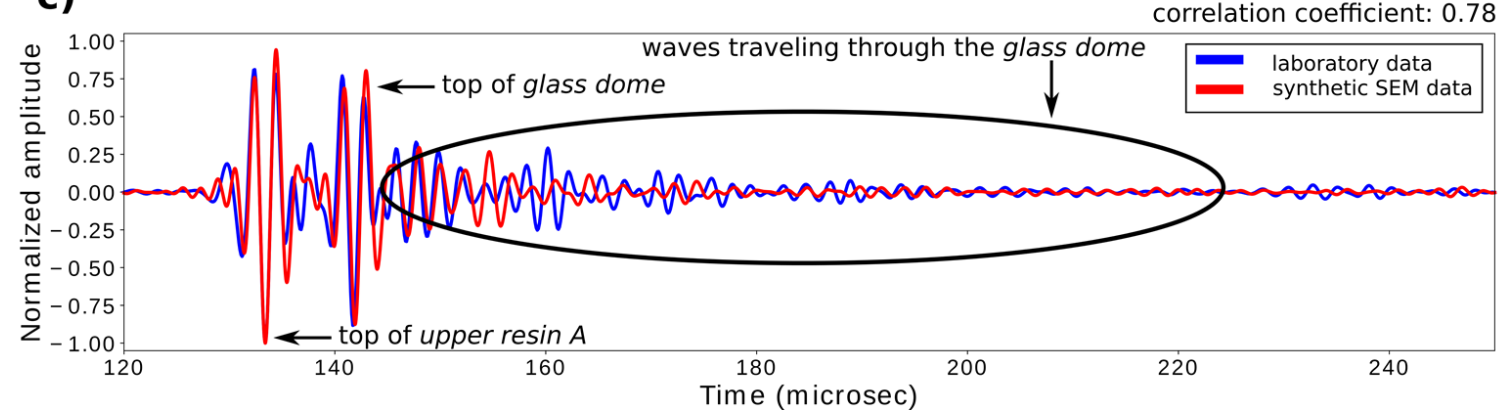

Figure 12. Comparison of offset laboratory traces with the corresponding synthetic SEM results: a) trace $F, b)$ trace $G, c)$ trace $H$.

\section{Discussion of the experimental and calibration procedure}

As shown above, we can accurately reconstruct the laboratory measurements for the WAVES model using the SEM with a regular grid. In general, a very good fit can be obtained between the laboratory zero-offset measurements and the numerical simulations, which is also highlighted by the very high correlation coefficients of at least 0.82 . Although the observed misfits are somewhat higher in the offset configuration, the values of the correlation coefficients $(0.78$ or higher $)$ still suggest a good reconstruction of the laboratory offset traces as well.

At this point, it is interesting to focus more specifically on the possible origins of the observed misfits and to evaluate as much as possible the associated uncertainties and the degree of significance of their impact on the misfits. In this sense, we would like to provide here a hierarchical list of uncertainties to which particular attention should be paid. By hierarchical list, we mean from the least to the most affecting uncertainty. Far from being exhaustive, this list may nevertheless be useful in future studies when laboratory experiments and numerical simulations are to be combined or compared. Three different factors can be distinguished: those related exclusively to the numerical simulations, those related exclusively to the experiments, and those related to both the experimental and the numerical aspects.

In the numerical simulations, the highly complex model geometry has been implemented 
using an equidistant grid. Of course, such a grid cannot explicitly honor the model discontinuities, especially in the case of the sides of the dome and the junctions of the different resin layer boundaries next to the flanks of the dome. Nevertheless, the good fit observed for the reflections from the curved top surface of the glass dome, in both zero-offset and offset configurations, provides a reassuring feedback on the current implementation of the model geometry. Therefore, we consider this effect to be minimal.

The reduced-scale experiments are of physical nature. Hence, similar to field data, our laboratory data still contain noise, although each signal was stacked several hundreds of times, in order to reduce the relative amplitude of any spurious incoherent noise and to enhance the signal-tonoise ratio. The presence of noise may be problematic mainly in offset configurations where signals can have very low amplitudes (Figures 11 and 12). In this case, it may be useful to post-process laboratory data by applying appropriate denoising methods (Traoré et al., 2017a, 2017b), after identifying the resulting noise characteristics. Nevertheless, the noise is far from being the main issue we have to face during the experiments, and we consider the impact of the noise on the quality of the fit between numerical simulations and laboratory data to be small.

On the contrary, two factors appear to have much a greater impact on the quality of the fit between numerical simulations and laboratory data: the numerical implementation of the source characteristics and that of the material properties. As already pointed out in Solymosi et al. (2018), the implementation of the source characteristics used here does not describe perfectly the real directivity of the transducer and its full waveform (since the later arrivals of the wavelet are poorly reconstructed). Indeed, although the strongly energetic main lobe of the source transducer is very well restored (as it can be seen from the good fit between numerical and experimental data in zero-offset configuration), the lower-energy secondary lobes are poorly recovered by the inversion strategy proposed by Solymosi et al. (2018). In other words, this strategy seems to be inadequate in case of low signal-to-noise ratios. Therefore, further work must be done both from a theoretical point of view in order to improve the strategy, and from the experimental side to maximize the signal-to-noise ratio in the directivity measurements.

Although the precision of the laboratory measurements has been significantly improved compared to Solymosi et al. (2018), thanks to an a posteriori control of the transducer movements, there is still a high uncertainty in the initial transducer positions before the data acquisition, and this appears to have a major impact on the quality of the fit between numerical simulations and laboratory data, in particular for offset configurations. This uncertainty $( \pm 0.5 \mathrm{~mm})$ has to be definitely investigated in detail and decreased in future works, probably with the help of metrologists (e.g., Pitre et al., 2017).

Before simulating wave propagation for the complex WAVES model, we had performed a numerical calibration of the material properties, consisting of adjusting the velocity and attenuation values, such that there is a perfect fit between the chosen laboratory traces and the corresponding numerical ones for the zero-offset configuration. This preliminary and quite common procedure is necessary as the uncertainties are quite high in the laboratory characterization of the material properties, especially for the attenuation fields. However, we could observe that the obtained material properties are significantly influenced by the position of the transducer, and hence by the zone of the 
model which is illuminated by the transducer. They implicitly bear the signature of wave phenomena (e.g., mild focusing/defocusing, diffraction). In other words, the calibrated properties are rather effective/apparent than intrinsic, therefore they do not provide perfect results for all the comparisons between laboratory and numerical data. Including offset data in the calibration procedure from the beginning would have led to a different set of material properties, but it is not clear whether these properties would have produced a lower global misfit, since wave focusing/defocusing and diffraction by $3 \mathrm{D}$ curved structures are much more significant in the offset than in the zero-offset configuration. In future works, it would be particularly worthwhile to understand better this attenuation issue, both from theoretical and experimental aspects. Moreover, the relevancy of the calibration procedure and that of assuming constant Q-factors with frequency (as usually considered in seismics/seismology) in numerical algorithms should also be investigated.

At this point, one last question should be raised : has the reduced-scale model been perfectly manufactured according to the initial 3D plans? Indeed, it was highly challenging to find the optimal process to manufacture such a complex model and to ensure a perfect contact between the different material layers. A good way to check the quality of both the model and our "imperfect" experimental framework can be to apply reverse-time migration (RTM) to the laboratory data.

\section{REVERSE-TIME MIGRATION OF THE LABORATORY DATA AS AN ADDITIONAL TOOL TO VALIDATE THE EXPERIMENTAL FRAMEWORK}

As shown above, the interpretation of the laboratory data for the WAVES model without the necessary post-processing is challenging in the time domain, as for instance the late arrivals corresponding to ray paths through the dome could not be individually interpreted due to the complex geometry. Therefore, accurate reconstruction of the model geometry is crucial before interpreting the measured data. This geometric reconstruction is usually ensured by different seismic migration algorithms in an operational context. Depending on the vertical and lateral heterogeneities, and the expected maximal tilt angle of the discontinuities, different algorithms have been developed (e.g., Yilmaz, 1987, Virieux and Operto 2009). Here we use a standard seismic imaging technique, reversetime migration (RTM). RTM is based on the imaging principle (Claerbout, 1971), and it is capable to reconstruct the reflectors related to reflection coefficient contrasts, but it cannot determine the real amplitude of these reflectors (Zhang et al., 2003, Robein 2010). Our goal with applying RTM to the laboratory data sets is to validate the framework described above. Because RTM yields an image in the depth domain, if the true material properties are used and if our framework is correct overall (despite the uncertainties in the laboratory measurements, the characterization of the material properties, the numerical implementation of the transducer characteristics and the model geometry), the resulting RTM data set should strongly resemble the known model geometry.

\section{Brief description of the method}

RTM is based on seismic reciprocity, which exploits the time-invariant property of the wave equation for a non-attenuating medium. Namely, this property makes it possible to record a wavefield in the time domain, and then focus back the wavefield at the source position by propagating back in time the registration at the original receiver position (in a last in first out manner). In the context of time-reversed acoustics, seismic reciprocity was first used by Baysal et al. (1983), Mc Mechan (1983) and Whitmore (1983), and later by Fink (1999). 
Figure 13 shows the workflow of the applied RTM. First, offset laboratory data are acquired along the center line of the model in the x-direction (dashed line in Figure 1 middle left, and along the section shown in Figure 1e). To ensure the illumination of the physical model, we use three source tilt angles $\left(19.7 \pm 0.1^{\circ}, 25.1 \pm 0.1^{\circ}\right.$, and $\left.30.0 \pm 0.1^{\circ}\right)$ and record multiple acquisition lines with a source spacing of $20.000 \pm 0.005 \mathrm{~mm}$ and a receiver spacing of $0.500 \pm 0.005 \mathrm{~mm}$. These values correspond to a source spacing of $400.0 \pm 0.1 \mathrm{~m}$ and a receiver spacing of $10.0 \pm 0.1 \mathrm{~m}$ at seismic scale. Altogether 17 642 registrations on 42 survey lines are measured. Second, forward simulations are computed, using the same acquisition geometry as for the laboratory measurements. We performed the simulations with the 2D version of the SPECFEM software package to save computational cost, considering also the fact that RTM is mostly suitable to reconstruct discontinuities, but not well-suited for quantitative amplitude analysis. The geometry is implemented with a non-structured 2D quadrangular mesh in Cubit/Trelis (Blacker, 1994). The target element size of the mesh is $1.1 \mathrm{~mm}$, and we obtain approximately 73000 elements, considering the water above the model as well. The material properties are considered to be identical to the 3D case presented above. Following the forward simulations, the differences between the recorded and simulated registrations are computed for each receiver. These differences provide the source-time function of the so-called adjoint wavefield in the third step (e.g., Tromp et al., 2005, Virieux and Operto, 2009). The third step consists of two simulations at the same time, a forward simulation (identical to the second step) and the adjoint simulation, which means the forward propagation of the adjoint wavefield in time from the original receiver points of Steps 1 and 2.

Because the resin layers of the model have significant attenuation, it has to be taken into account in the calculations as well. We use the technique of parsimonious disk storage (also known as optimal checkpointing) of the forward wavefield (Komatitsch et al., 2016). As part of this strategy, the so-called checkpoint/restart files are saved to the disk during the forward simulation in Step 2. These files contain the displacement components and are typically stored for every few hundred time steps. Then in the third step, the forward simulation is not run backward in time which would be numerically unstable, but rather forward, using the checkpoint/restart files. At any time, the simulation is run forward in time using the corresponding restart file, read back from the disk. Because the simulations are always conducted forward in time, they are always stable, even in the case of attenuating materials. 


\section{$1^{\text {st }}$ step}

\section{Laboratory measurements:}

- offset configuration

- different source positions

- recording pressure

at near- and far-offsets

\section{$2^{\text {nd }}$ step}

Forward simulation:

- same configuration

as the laboratory measurements

- considering the measured material properties (including attenuation)
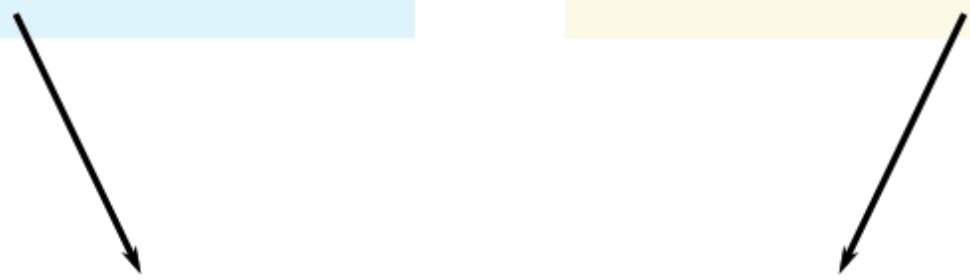

Compute the difference of the measured and simulated registrations (i.e. adjoint sources)

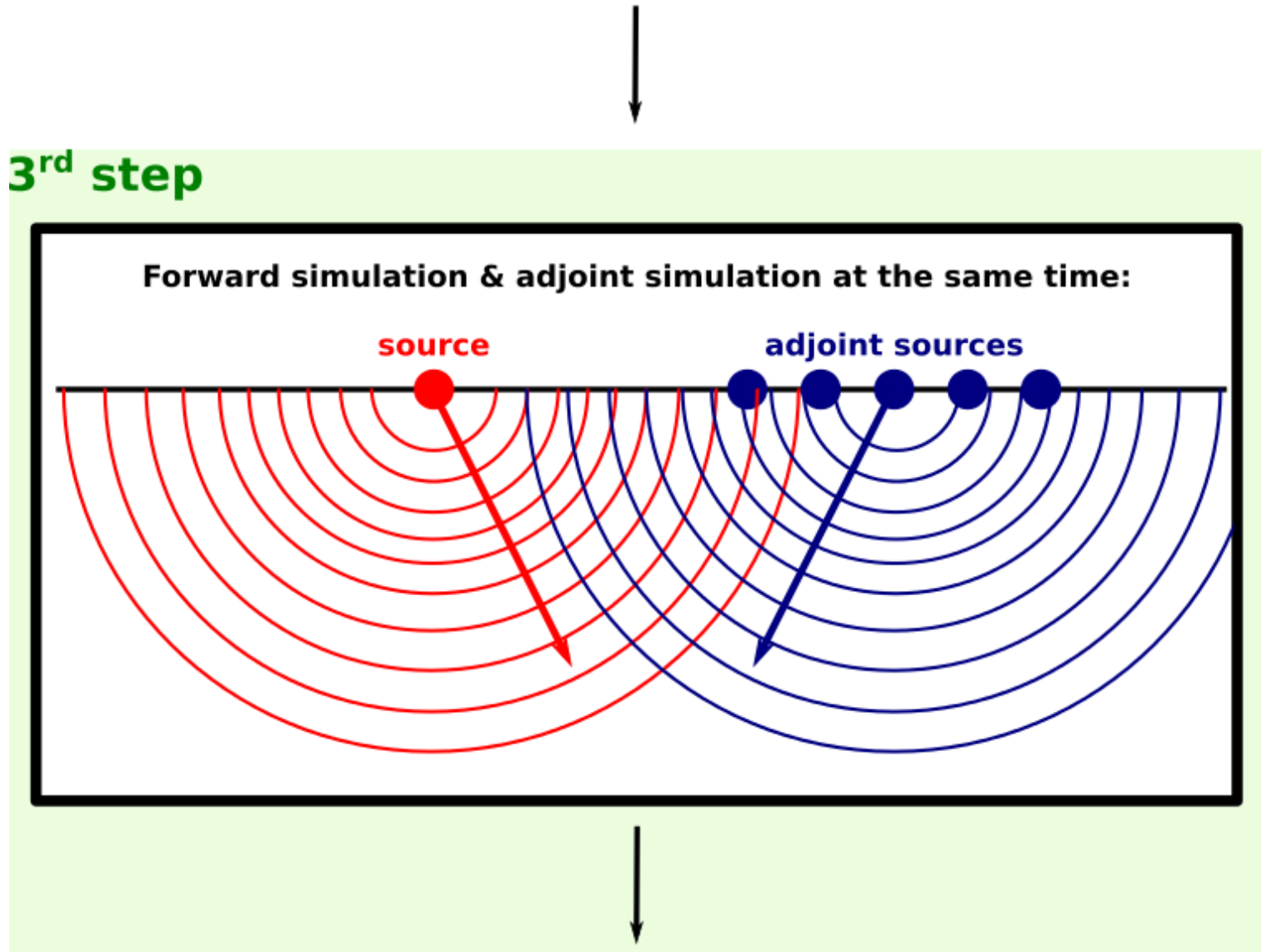

Output: correlation of the forward wavefield with the adjoint field

Figure 13. RTM workflow.

We use a smoothed version of the "real" model, as is general practice in seismic exploration. Here the term real refers to the best knowledge we have about the physical model, i.e., provided in the 3D geometry files of the model which were also used for the manufacturing. Hence, this does not take into account the possible misfits between the assumed (provided by these files) and actual real geometry. In addition, a Gaussian filter with a kernel size of $12 \times 12 \mathrm{~mm}^{2}$ (corresponding to 240x240 
$\mathrm{m}^{2}$ at seismic scale) is applied to each material property $\left(V_{P}, V_{S}, \rho, Q_{P}, Q_{S}\right)$ to obtain a smooth model (Figure 14). Figure 15a shows a 3D laboratory trace and the corresponding 2D simulated trace using the smoothed geometry. The source was positioned at $\mathbf{x}=19.9 \pm 0.5 \mathrm{~mm}$, in the center line in $\mathbf{y}-$ direction, and $100.0 \pm 0.5 \mathrm{~mm}$ above the model. The receiver was located at $\mathbf{x}=79.8 \pm 0.5 \mathrm{~mm}$, in the center line in y-direction, and $100.0 \pm 0.5 \mathrm{~mm}$ above the model. Figure $15 \mathrm{~b}$ illustrates the difference between the measured and simulated traces, i.e., the adjoint trace. We can clearly see that the adjoint trace contains information mainly about the laboratory measurements for the late arrivals after 245 $\mu \mathrm{s}$. These events correspond to the ray paths traveling through the glass dome. Thanks to the preservation of the laboratory information, the interpretation of the final RTM results is straightforward, as any high correlation value corresponds to the information on the geometry that has been measured during the laboratory experiments (and unrelated to to the numerical simulations for the smooth model in Figure 14).

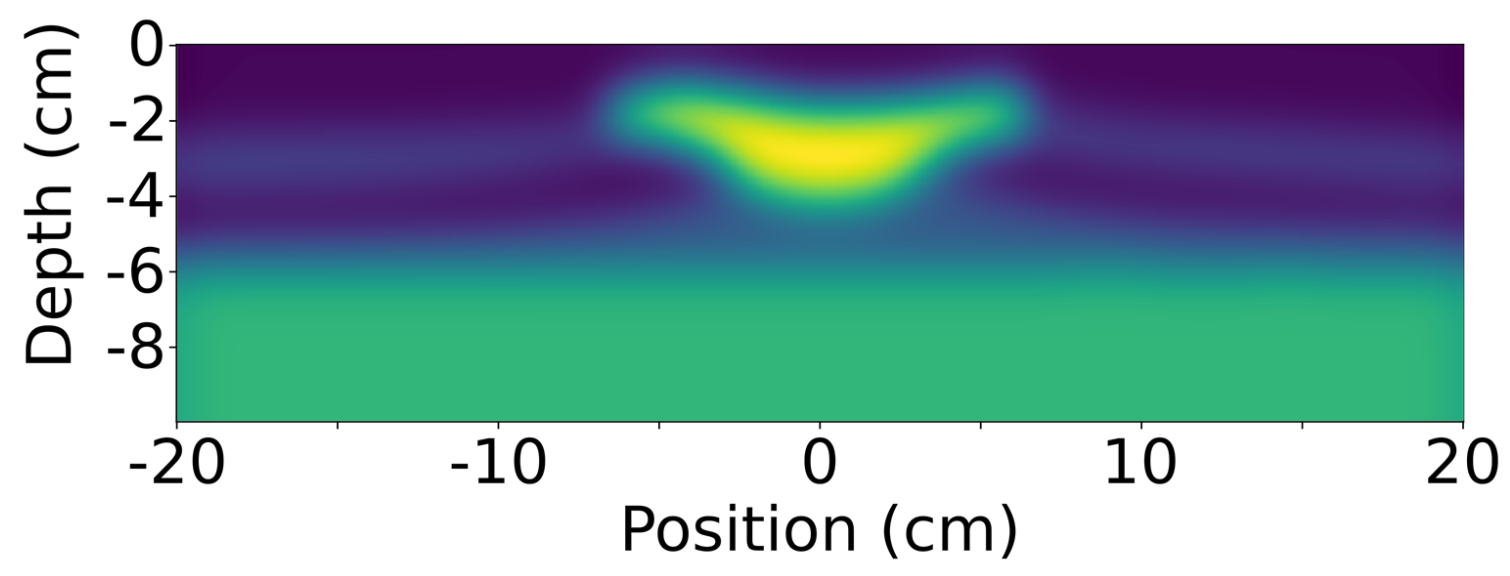

Density $\left(\mathrm{kg} / \mathrm{m}^{3}\right)$

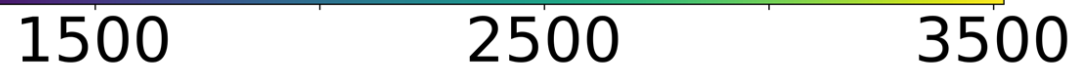

Figure 14. The smoothed density model used for the RTM calculations.
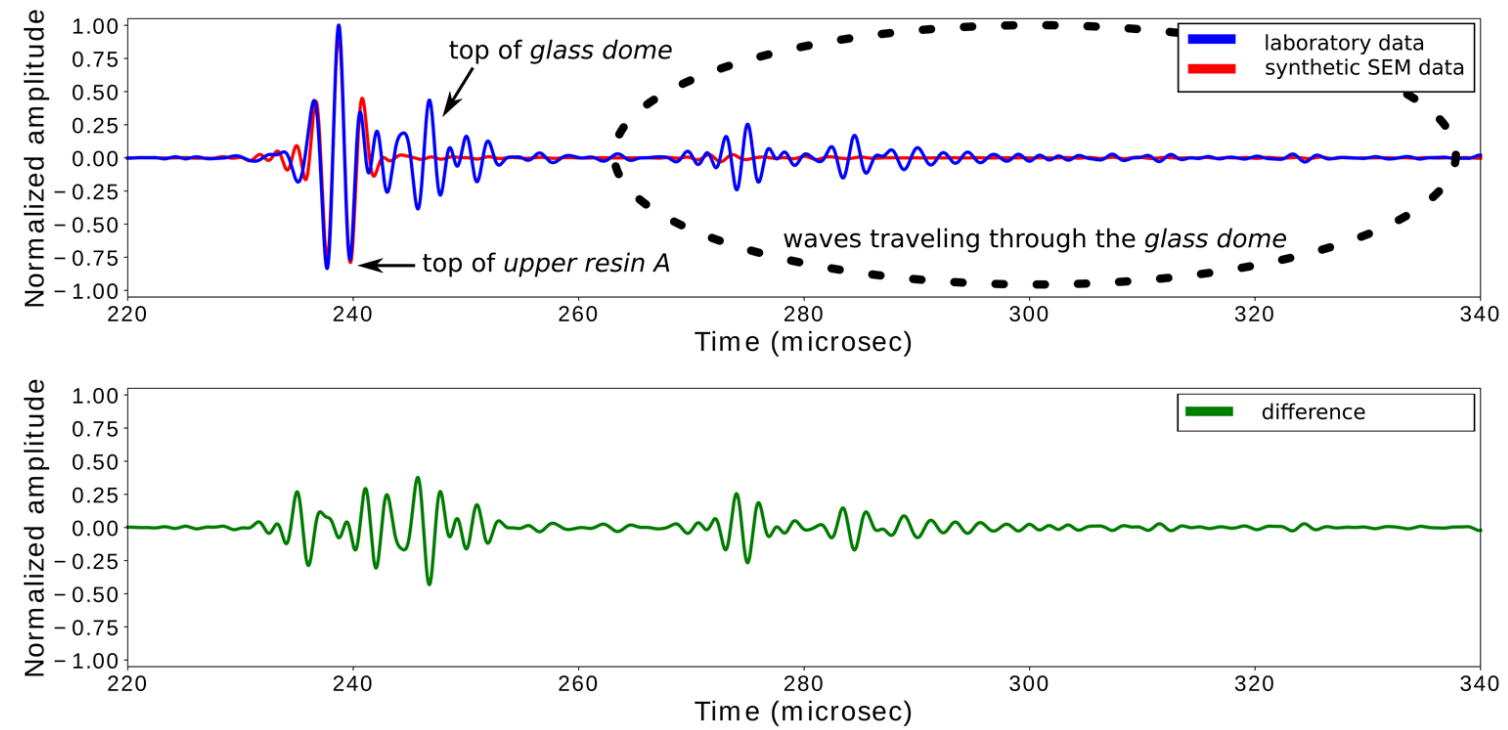

Figure 15. Illustration of the RTM calculations for the smoothed model, as shown in Figure 14. Top: laboratory trace (blue) and 2D simulated trace (red), bottom: difference (i.e. adjoint source). 
In RTM, the correlation of the forward and adjoint wavefields is calculated in the third step (Figure 13). However, as there is a strong connection between the full-waveform inversion (FWI) and RTM (e.g., Mora, 1988, Tromp et al., 2005), sensitivity kernels can also be defined for RTM and used similar to FWI for the imaging and geologic interpretation of the subsurface (e.g., Virieux and Operto, 2009, Zhu et al., 2009). In this case, some derivatives of the cross-correlated wavefields are used. These derivatives (also known as sensitivity kernels) depend on the formulation of the wave equation. We note that in practice there is no one formulation to be chosen in any case, but rather it depends on the actual geologic setup and the recorded data itself (e.g., seismic exploration, global seismology, etc.) (e.g., Zhu et al., 2009). We calculate the two most common formulations here, first, the density $\rho$, bulk modulus $\kappa$, shear modulus $\mu$ formulation, then the impedance $\rho^{\prime}$, compressional-wave speed $\alpha$, shear-wave speed $\beta$ formulation. An extensive explanation of the calculations can be found, for example, in Liu and Tromp (2006), Tromp et al. (2008), Zhu et al. (2009), Douma et al. (2010), and Luo et al. (2013). Here we present only the sensitivity kernels which are used in this paper:

$$
\begin{gathered}
K_{\rho}(x)=\int_{0}^{T} \quad \rho(x) \partial_{t} s^{*}(x, T-t) \cdot \partial_{t} s(x, t) d t,(1) \\
K_{\kappa}(x)=-\int_{0}^{T} \kappa(x)\left[\nabla \cdot s^{*}(x, T-t)\right][\nabla \cdot s(x, t)] d t,(2) \\
K_{\mu}(x)=-\int_{0}^{T} 2 \mu(x) D^{*}(x, T-t): D(x, t) d t,(3)
\end{gathered}
$$

namely, the density $K_{\rho}$, bulk modulus $K_{\kappa}$, and shear modulus $K_{\mu}$ kernels, respectively, where $\boldsymbol{x}$ is the spatial coordinate, the integral is over the time $(t=0 . . T), s$ is the displacement, $s^{*}$ is the adjoint source, $D=\frac{1}{2}\left[\nabla s+(\nabla s)^{T}\right]-\frac{1}{3}(\nabla \cdot s) I$ and $D *$ are the traceless strain deviator and its adjoint, respectively (Tromp et al., 2005), and $\boldsymbol{I}$ is the identity matrix. The definition of the second set of sensitivity kernels are based on equations 1-3:

$$
\begin{gathered}
K_{\rho^{\prime}}=K_{\rho}+K_{\kappa}+K_{\mu},(4) \\
K_{\alpha}=2 K_{\kappa}\left(1+\frac{4}{3} \frac{\mu}{\kappa}\right),(5) \\
K_{\beta}=2\left(K_{\mu}-\frac{4}{3} \frac{\mu}{\kappa} K_{\kappa}\right)(6)
\end{gathered}
$$

for the impedance $K_{\rho}^{\prime}$, compressional-wave speed $K_{\alpha}$, and shear-wave speed $K_{\beta}$ kernels, respectively.

\section{RTM results}

Figure 16 shows the resulting sensitivity kernels obtained with the RTM. From the three kernels of the $\mathrm{K}_{\rho}-\mathrm{K}_{\kappa}-\mathrm{K}_{\mu}$ formulation, $\mathrm{K}_{\kappa}$ can be interpreted most easily (Figure 16b). Although this kernel shows artifacts as well, the different discontinuities of the model can be interpreted easier than for $K_{\rho}$ or $K_{\mu}$. As pointed out by Zhu et al. (2009), RTM images usually suffer from artifacts, due to diving waves, head waves, and backscattered waves. They suggest relying on the impedance kernel $\mathrm{K}_{\rho}$, which is the sum of $\mathrm{K}_{\rho}, \mathrm{K}_{\kappa}$, and $\mathrm{K}_{\mu}$ (equation 4), because the low-frequency artifacts cancel each other during the summation. This observation is confirmed for the WAVES model, as observed in 
Figure $16 \mathrm{~d}$. Considering either $\mathrm{K}_{\kappa}$ or $\mathrm{K}_{\rho^{\prime}}$, we can easily interpret the top of the physical model at a 0 $\mathrm{cm}$ depth. The other discontinuities of the model can also be interpreted, especially in the middle of the sections, where the model is better illuminated than the sides. Furthermore, there is a difference between the coherence of these discontinuities on the left and right sides of each kernel. That is because the source was always located on the left side during the laboratory experiments, therefore the receiver could not image beyond the source position. Last but not least, we see a proper imaging of all sides of the glass dome, also on the bottom. The top of the aluminum at a depth of $5.5 \mathrm{~cm}$ can also be interpreted at its known position.

In order to validate the resulting RTM images from the perspective of the spatial dimensions of the geometry, Figure 17 shows $\mathrm{K}_{\rho^{\prime}}$ superimposed to the color-coded known reference geometry. From a qualitative viewpoint, we can see an almost perfect fit between the sensitivity kernels and the original geometry for each domain of the model. 
a)

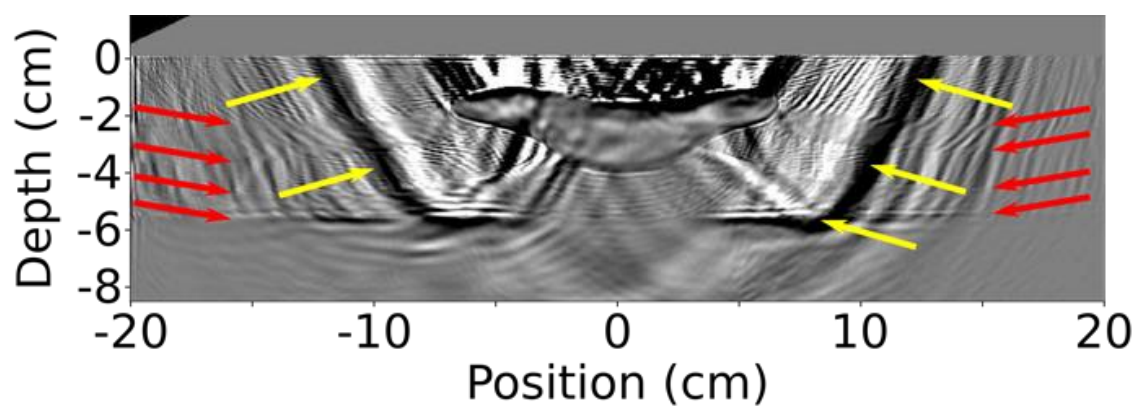

b)

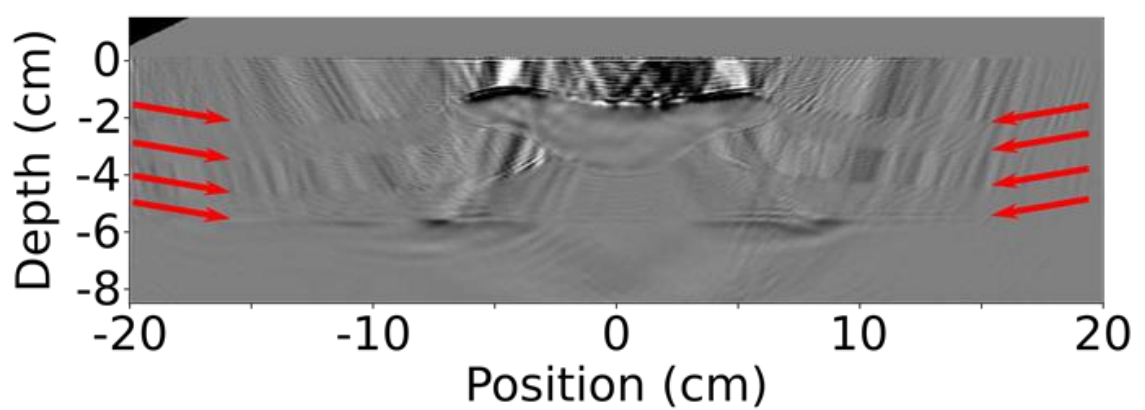

c)

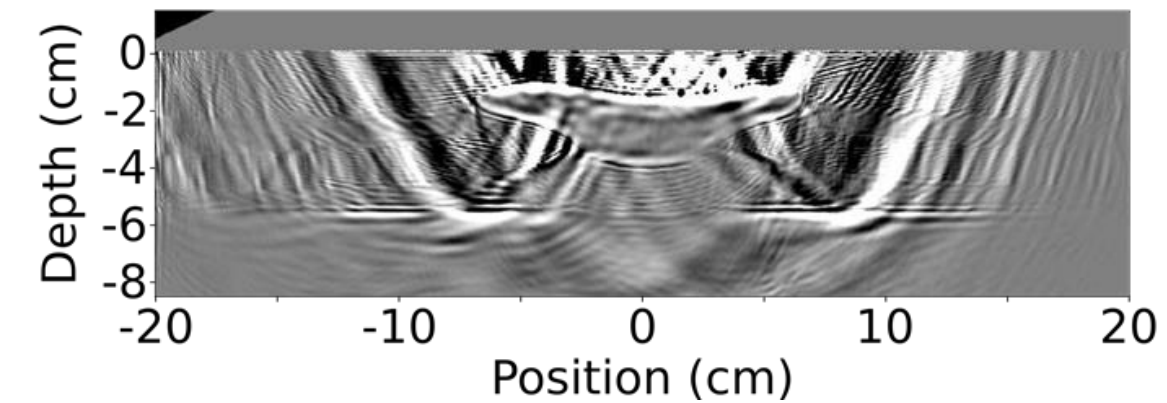

d)

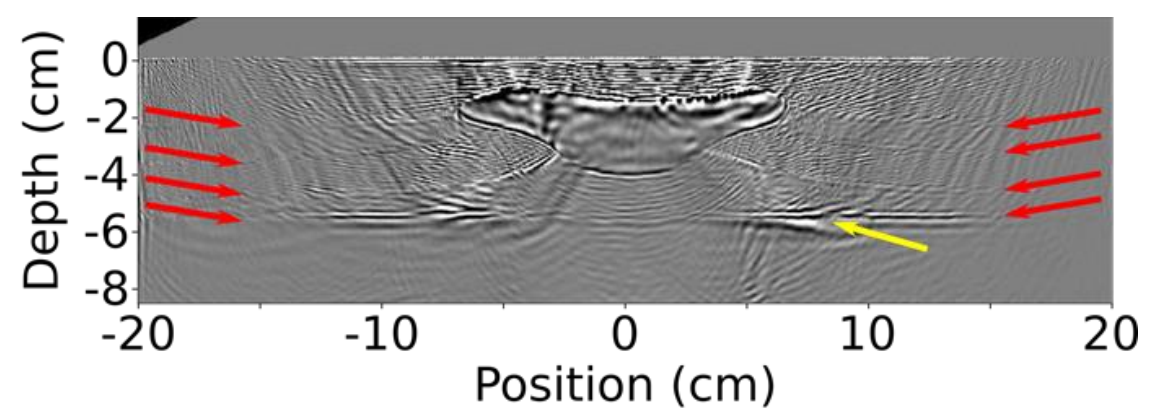

e)

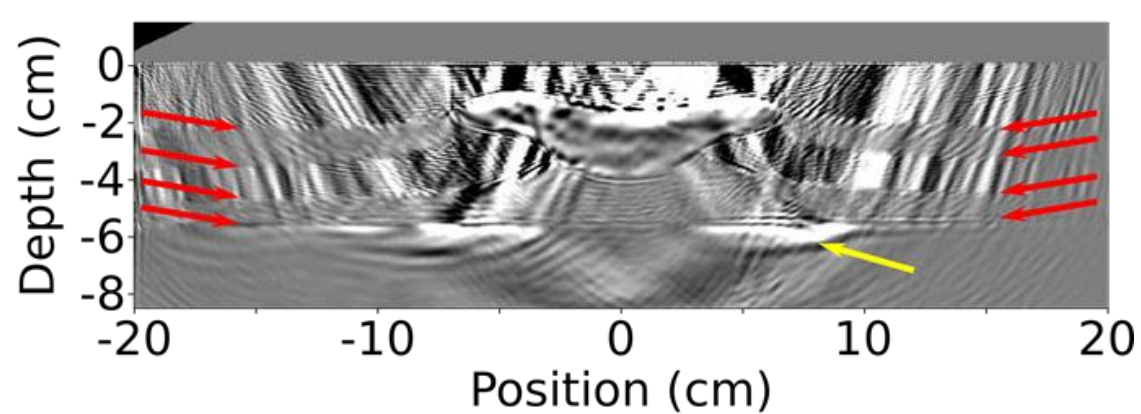

f)

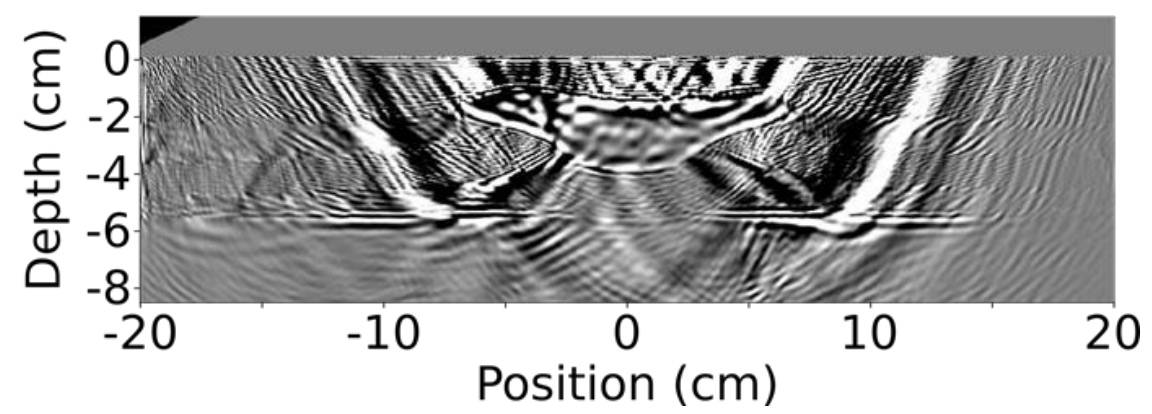

Figure 16. Sensitivity kernels obtained with RTM: a) density kernel $\mathrm{K}_{\rho}$, b) bulk modulus kernel $\mathrm{K}_{\kappa}$, c) shear modulus kernel $\mathrm{K}_{\mu}$, d) impedance kernel $\mathrm{K}_{\rho}$, e) compressional-wave speed kernel $\mathrm{K}_{\alpha}$, and $\mathrm{f}$ ) shear-wave speed kernel $\mathrm{K}_{\beta}$. Yellow arrows: artifacts, red arrows: known model discontinuities. 

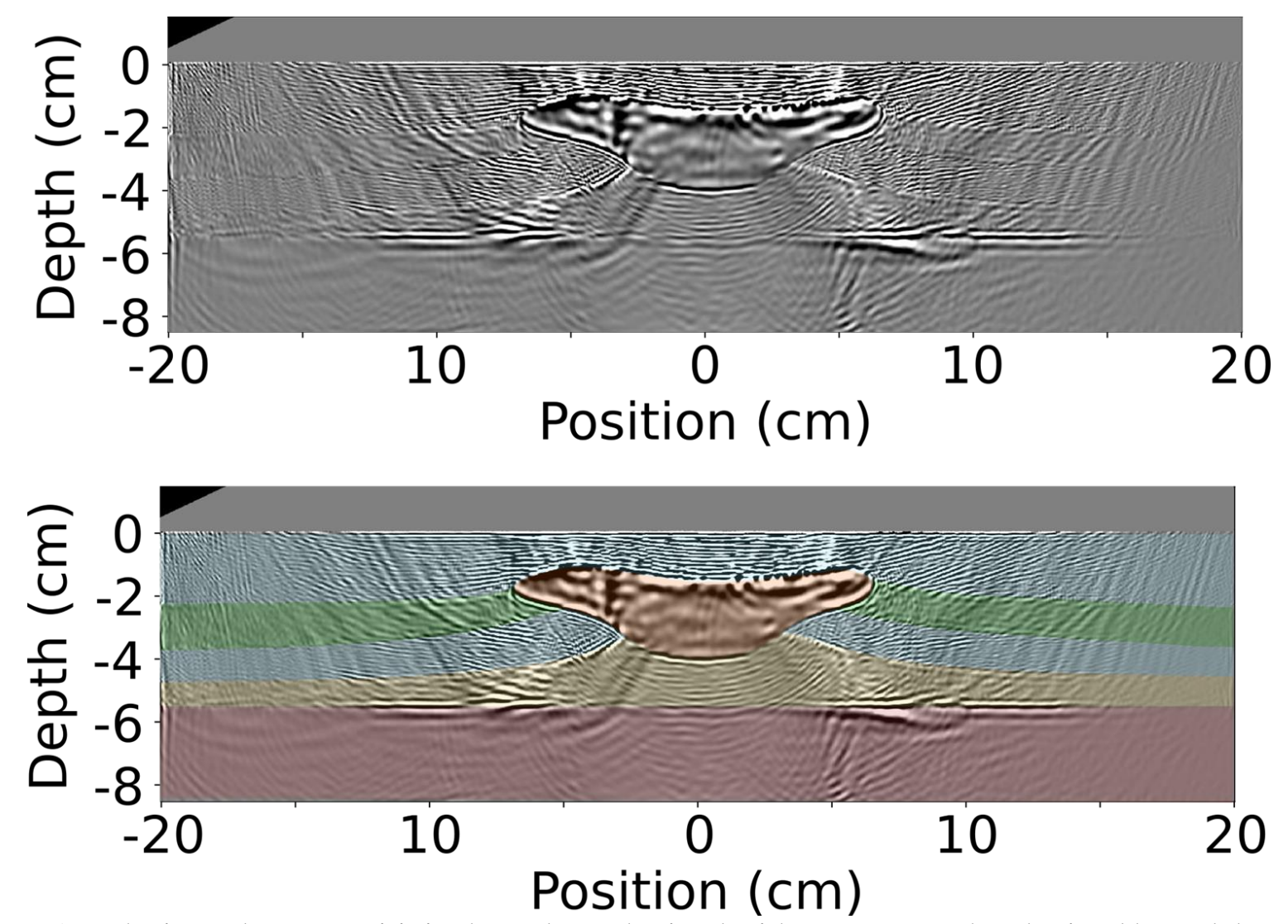

Figure 17. The impedance sensitivity kernel $\mathrm{K}_{\rho^{\prime}}$ obtained with RTM. Top: the obtained kernel, bottom: the obtained kernel with an overlay of the original geometry (in color).

\section{Discussion of the RTM results}

As shown above, we can successfully visualize the inner geometry of the WAVES model with a 2D RTM, using the 3D laboratory data sets. We found that the bulk modulus and the impedance kernels are the most suitable for the interpretation of these data sets. The known discontinuities of the model are almost all restored. The top surface of the physical model, as well as all sides of the glass dome, and the top of the aluminum are entirely recovered. The other discontinuities are wellreconstructed in the central positions, somewhat less coherently at greater depths, or sometimes next to the dome. Artifacts can also be interpreted on the RTM images, which are partly expected with the RTM, due to diving waves, head waves, and backscattered waves. In accordance with the literature (e.g. Zhu et al., 2009), the impedance kernel suffers from fewer artifacts than, for example, the bulk modulus kernel.

Although the comparison of RTM images with the original geometry shows an almost perfect overlap, we need to keep in mind the non-equivalence of the 2D and 3D for a complex geometry like the WAVES model. The images could be improved by re-performing RTM in 3D where sources and receivers were deployed out of the current acquisition plane, as well as by using more sources with a smaller source spacing. Nevertheless, our main goal with applying RTM to the laboratory data was to provide an additional tool to validate the experimental framework for complex models such as the WAVES model, which we can consider achieved here.

\section{CONCLUSIONS}

The goal of this work was to provide a global overview of the framework to be implemented 
to obtain high-quality laboratory data in the context of offshore seismics, regardless of the geological environment. To illustrate it, we have relied on a reduced-scale model representing a 3D complexshaped salt body buried in sedimentary layers with curved surfaces. The model is made of a specific crystal, different resins with aluminum and silicon powders, and aluminum. Zero-offset and offset reflection data have been acquired for this model in a water tank, using a conventional pulse-echo technique. Following previous works, the framework uses cross-validation of laboratory and numerical data, to point out the necessary improvements in both the experimental and numerical parts by reducing the measurements uncertainties and improving the numerical implementations, respectively. We have thus set up a hierarchical list of points (from the least to the most affecting the quality of the numerical simulations and the experimental data) to which particular attention should be paid to allow laboratory experiments to be very efficient tools for seismic exploration. For instance, inaccuracies in the initial position of the transducers, numerical implementation of the source directivity pattern, and uncertainties in the estimation of the media properties (especially, for attenuation) may have a major effect on the data and simulations quality. These issues may be solved with the help of metrologists. On the contrary, the quality of the meshing of the complex model and the presence of noise, which can be reduced by using proper signal processing techniques, introduce minimal adverse effects. Finally, the quality of both the complex reduced-scale model and the global framework proposed here has been successfully validated by successfully applying reverse-time migration to the laboratory data. Benefiting from the on-going technological advances, we believe that in the future laboratory experiments can play a major role to support the field experiments and numerical modeling, or to investigate some of the current challenges of seismic imaging in terms of, e.g., acquisition design or benchmarking of new imaging techniques at a low cost and in a versatile way.

\section{ACKNOWLEDGMENTS}

This paper is dedicated to our colleague, D. Komatitsch who passed away on 21 January 2019. The authors thank G. Machado for his valuable help with the digitization of the 3D geometry files, as well as R. Guillermin, G. Rabau, V. Long, E. Debieu, P. Lasaygues, and P. Sanchez for tackling the challenges of the laboratory experiments with us. TheWAVES model could not become a reality without the great and precise work of La Fonderie de Verre (O. Fonderflick) and VN Composites (Nicolas and J.-P. Tronquoy). They continuously consulted us before and during the building process of the physical model to properly translate our various ideas and are warmly thanked here. This work received funding from the European Union Horizon 2020 research and innovation program under the Marie Skłodowska-Curie grant agreement no. 641943. B. Ursin thanks the Norwegian Research Council and the industry partners of the GAMES consortium for financial support (grant no. 294404). We also thank CNRS for its financial support through the PICS BENCHIE project. This work was granted access to the French HPC resources of TGCC under allocation no. 2017-gen7165 made by GENCI. We also acknowledge the associate editor and the anonymous reviewer for their careful reading, helpful suggestions, and relevant questions, all of which greatly improved the paper.

\section{DATA AND MATERIALS AVAILABILITY}

Data associated with this research are available and can be obtained by contacting Nathalie FavrettoCristini.

\section{REFERENCES}


Baysal, E., D. D. Kosloff, and J. W. Sherwood, 1983, Reverse time migration: Geophysics, 48, 15141524.

Becker, T. S., D.-J. van Manen, C. M. Donahue, C. Bärlocher, N. Börsing, F. Broggini, T. Haag, J. O. A. Robertsson, D. R. Schmidt, S. A. Greenhalgh, and T. E. Blum, 2018, Immersive Wave Propagation Experimentation: Physical Implementation and One-Dimensional Acoustic Results: Physical Review $\mathrm{X}, \mathbf{8}, 031011$.

Blacker, T., 1994, CUBIT Mesh Generation Environment Users Manual, https://cubit.sandia.gov/public/13.2/help_manual/WebHelp/cubit_users_manual.html, accessed 08 October 2018.

Bretaudeau, F., D. Leparoux, O. Durand, and O. Abraham, 2011, Small-scale modeling of onshore seismic experiment: A tool to validate numerical modeling and seismic imaging methods: Geophysics, 76, no. 5, T101-T112.

Bretaudeau, F., R. Brossier, D. Leparoux, O. Abraham, and J. Virieux, 2013, 2D elastic full-waveform imaging of the near-surface: Application to synthetic and physical modelling data sets: Near Surface Geophysics, 11, 307-316.

Campman, X. H., K. van Wijk, J. A. Scales, and G. C. Herman, 2005, Imaging and suppressing nearreceiver scattered surface waves: Geophysics, 70, no. 2, V21-V29.

Chai, X., S. Wang, J. Wei, J. Li, and H. Yin, 2015, Reflectivity inversion for attenuated seismic data: Physical modeling and field data experiments: Geophysics, 81, no. 1, T11-T24.

Chang, C.-H., Y.-F. Chang, and P.-Y. Tseng, 2017, Azimuthal variation of converted-wave amplitude in a reservoir with vertically aligned fractures - a physical model study: Geophysical Prospecting, 65, 221-228.

Claerbout, J. F., 1971, Toward a unified theory of reflector mapping: Geophysics, 36, 467-481.

Cooper, J. K., D. C. Lawton, and G. F. Margrave, 2010, The wedge model revisited: A physical modeling experiment: Geophysics, 75, no. 2, T15-T21.

Douma, H., D. Yingst, I. Vasconcelos, and J. Tromp, 2010, On the connection between artifact filtering in reverse-time migration and adjoint tomography: Geophysics, 75, no. 6, S219-S223.

Ebrom, D. A., and J. A. McDonald, 1994, Seismic physical modeling: SEG.

Ekanem, A., J. Wei, X.-Y. Li, M. Chapman, and I. Main, 2013, P-wave attenuation anisotropy in fractured media: A seismic physical modelling study: Geophysical Prospecting, 61, 420-433.

Favretto-Anrès, N., and G. Rabau, 1997, Excitation of the Stoneley-Scholte wave at the boundary between an ideal fluid and a viscoelastic solid: Journal of Sound and Vibration, 203, 193-208. 
Favretto-Cristini, N., A. Tantsereva, P. Cristini, B. Ursin, D. Komatitsch, and A. M. Aizenberg, 2014, Numerical modeling of zero-offset laboratory data in a strong topographic environment : Results for a spectral-element method and a discretized Kirchhoff integral method: Earthquake Science, 27, 391399.

Favretto-Cristini, N., A. M. Aizenberg, B. Ursin, P. Cristini, and A. Tantsereva, 2017, Analysis of Wave Scattering from a Viscoelastic Layer with Complex Shape: Journal of Computational Acoustics, 25, 1750023-1-1750023-12.

Fichtner, A., 2010, Full seismic waveform modelling and inversion: Springer-Verlag.

Fink, M., 1999, Time-reversed acoustics: Scientific American, 281, 91-97.

Fleury, C., 2013, Increasing illumination and sensitivity of reverse-time migration with internal multiples: Geophysical Prospecting, 61, 891-906.

Hughes, T. J., 1987, The finite element method: linear static and dynamic finite element analysis: Prentice-Hall International Inc.

Jackson, M. P., and M. R. Hudec, 2017, Salt tectonics: Principles and practice: Cambridge University Press.

Jones, I. F., and I. Davison, 2014, Seismic imaging in and around salt bodies: Interpretation, 2, SL1SL20.

Komatitsch, D., and J.-P. Vilotte, 1998, The spectral element method: an efficient tool to simulate the seismic response of 2D and 3D geological structures: Bulletin of the Seismological Society of America, 88, 368-392.

Komatitsch, D., Z. Xie, E. Bozdag, E. Sales de Andrade, D. Peter, Q. Liu, and J. Tromp, 2016, Anelastic sensitivity kernels with parsimonious storage for adjoint tomography and full waveform inversion: Geophysical Journal International, 206, 1467-1478.

Liu, Q., and J. Tromp, 2006, Finite-frequency kernels based on adjoint methods: Bulletin of the Seismological Society of America, 96, 2383-2397.

Liu, Y., H. Hu, X.-B. Xie, Y. Zheng, and P. Li, 2015, Reverse-time migration of internal multiples for subsalt imaging: Geophysics, 80, no. 5, S175-S185.

Luo, Y., J. Tromp, B. Denel, and H. Calandra, 2013, 3D coupled acoustic-elastic migration with topography and bathymetry based on spectral-element and adjoint methods: Geophysics, 78, no. 4, S193-S202.

McMechan, G.A., 1983, Migration by extrapolation of time-dependent boundary values: Geophysical Prospecting, 31, 413-420. 
Mora, P., 1988, Elastic wavefield inversion of reflection and transmission data: Geophysics, 53, 750759.

Pageot, D., D. Leparoux, M. Le Feuvre, O. Durand, P. Côte, and Y. Capdeville, 2017, Improving the seismic small-scale modelling by comparison with numerical methods: Geophysical Journal International, 211, 637-649.

Peter, D., D. Komatitsch, Y. Luo, R. Martin, N. Le Goff, E. Casarotti, P. Le Loher, F. Magnoni, Q. Liu, C. Blitz, T. Nissen-Meyer, P. Basini, and J. Tromp, 2011, Forward and adjoint simulations of seismic wave propagation on fully unstructured hexahedral meshes: Geophysical Journal International, 186, 721-739.

Pitre, L., F. Sparasci, L. Risegari, C. Guianvarc'h, C. Martin, M. E. Himbert, M. D. Plimmer, A. Allard, B. Marty, P. G. Albo, and B. Gao, 2017, New measurement of the Boltzmann constant k by acoustic thermometry of helium-4 gas: Metrologia, 54, 856-873.

Robein, E., 2010, Seismic Imaging: A Review of the Techniques, their Principles, Merits and Limitations., EAGE Publications.

Roberts, A. W., R. W. Hobbs, M. Goldstein, M. Moorkamp, M. Jegen, and B. Heincke, 2016, Joint stochastic constraint of a large data set from a salt dome: Geophysics, 81, no. 2, ID1-ID24.

Robertsson, J. O. A., B. Bednar, J. Blanch, C. Kostov, and D.-J. van Manen, 2007, Introduction to the supplement on seismic modeling with applications to acquisition, processing, and interpretation: Geophysics, 72, no. 5, SM1-SM4.

Shepherd, J. F., and C. R. Johnson, 2008, Hexahedral mesh generation constraints: Engineering with Computers, 24, 195-213.

Solymosi, B., N. Favretto-Cristini, V. Monteiller, D. Komatitsch, P. Cristini, B. Arntsen, and B. Ursin, 2018, How to adapt numerical simulation of wave propagation and ultrasonic laboratory experiments to be comparable - A case study for a complex topographic model: Geophysics, 83, no. 4, T195-T207.

Staten, M. L., R. A. Kerr, S. J. Owen, T. D. Blacker, M. Stupazzini, and K. Shimada, 2010, Unconstrained plastering - Hexahedral mesh generation via advancing-front geometry decomposition: International Journal for Numerical Methods in Engineering, 81, 135-171.

Stewart, R. R., N. Dyaur, B. Omoboya, J. De Figueiredo, M. Willis, and S. Sil, 2012, Physical modeling of anisotropic domains: Ultrasonic imaging of laser-etched fractures in glass: Geophysics, 78, no. 1, D11-D19.

Tantsereva, A., B. Ursin, N. Favretto-Cristini, P. Cristini, and A. M. Aizenberg, 2014a, Numerical modeling of 3D zero-offset laboratory data by a discretized Kirchhoff integral method: Geophysics, 79, no. 2, T77-T90.

Tantsereva, A., B. Ursin, N. Favretto-Cristini, P. Cristini, and A. M. Aizenberg, 2014b, Erratum to: 
"Numerical modeling of 3D zero-offset laboratory data by a discretized Kirchhoff integral method": Geophysics, 79, no. 5, Y3-Y4.

Tari, G., J. Molnar, and P. Ashton, 2003, Examples of salt tectonics from West Africa: a comparative approach: Geological Society, London, Special Publications, 207, 85-104.

Traoré, O. I., L. Pantera, N. Favretto-Cristini, P. Cristini, S. Viguier-Pla, and Ph. Vieu, 2017a, Structure analysis and denoising using Singular Spectrum Analysis: application to acoustic emission signals from nuclear safety experiments: Measurement, 104, 78-88.

Traoré, O. I., N. Favretto-Cristini, L. Pantera, P. Cristini, S. Viguier-Pla, and Ph. Vieu, 2017b, Which methods and strategies to cope with noise complexity for an effective interpretation of acoustic emission signals in noisy nuclear environment ?: Acta Acustica united with Acustica 103, 903-916.

Tromp, J., C. Tape, and Q. Liu, 2005, Seismic tomography, adjoint methods, time reversal and banana-doughnut kernels: Geophysical Journal International, 160, 195-216.

Tromp, J., D. Komatitsch, and Q. Liu, 2008, Spectral-element and adjoint methods in seismology: Communications in Computational Physics, 3, 1-32.

Vasmel, M., J. O. A. Robertsson, D.-J. van Manen, and A. Curtis, 2013, Immersive experimentation in a wave propagation laboratory: The Journal of the Acoustical Society of America, 134, EL492EL498.

Virieux, J., H. Calandra, and R.-É. Plessix, 2011, A review of the spectral, pseudo-spectral, finitedifference and finite-element modelling techniques for geophysical imaging: Geophysical Prospecting, 59, 794-813.

Virieux, J., and S. Operto, 2009, An overview of full-waveform inversion in exploration geophysics: Geophysics, 74, no. 6, WCC1-WCC26.

Whitmore, N.D., 1983, Iterative depth migration by backward time propagation: : 53rd Annual International Meeting, SEG, Expanded Abstracts, 827- 830.

Xu, C., B. Di, and J. Wei, 2016, A physical modeling study of seismic features of karst cave reservoirs in the Tarim Basin, China: Geophysics, 81, no. 1, B31-B41.

Yilmaz, O., 1987, Seismic Data Processing: SEG.

Zhang, Y., G. Zhang, and N. Bleistein, 2003, True amplitude wave equation migration arising from true amplitude one-way wave equations: Inverse Problems, 19, 1113-1138.

Zhou, H.-W., H. Hu, Z. Zou, Y. Wo, and O. Youn, 2018, Reverse time migration: A prospect of seismic imaging methodology: Earth-Science Reviews, 179, 207-227.

Zhu, H., Y. Luo, T. Nissen-Meyer, C. Morency, and J. Tromp, 2009, Elastic imaging and time-lapse 
migration based on adjoint methods: Geophysics, 74, no. 6, WCA167-WCA177. 